\title{
Specific and redundant activities of ETV1 and ETV4 in prostate cancer aggressiveness revealed by co-overexpression cellular contexts
}

\author{
Diana Mesquita ${ }^{1}$, João D. Barros-Silva ${ }^{1}$, Joana Santos ${ }^{1}$, Rolf I. Skotheim ${ }^{2,3}$, \\ Ragnhild A. Lothe ${ }^{2,3}$, Paula Paulo ${ }^{1,2,3}$ and Manuel R. Teixeira ${ }^{1,3,4}$ \\ ${ }^{1}$ Department of Genetics and Cancer Genetics Group - CI-IPOP, Portuguese Oncology Institute, Porto, Portugal \\ ${ }^{2}$ Department of Cancer Prevention, Institute for Cancer Research, The Norwegian Radium Hospital, Oslo University Hospital, \\ Nydalen, Oslo, Norway \\ ${ }^{3}$ Centre for Cancer Biomedicine, Faculty of Medicine, University of Oslo, Oslo, Norway \\ ${ }^{4}$ Department of Pathology and Molecular Immunology, Institute of Biomedical Sciences Abel Salazar (ICBAS), \\ University of Porto, Portugal \\ Correspondence to: \\ Manuel R. Teixeira, e-mail: manuel.teixeira@ipoporto.min-saude.pt \\ Keywords: prostate cancer, ETV1 and ETV4 co-overexpression, target genes, oncogenic role, PEA3-positive tumors \\ Received: July 25, 2014 \\ Accepted: December 5, 2014 \\ Published: February 14, 2015
}

\section{ABSTRACT}

Genomic rearrangements involving ETS transcription factors are found in $50-70 \%$ of prostate carcinomas. While the large majority of the rearrangements involve ERG, around $10 \%$ involve members of the PEA3 subfamily (ETV1, ETV4 and ETV5). Using a panel of prostate cancer cell lines we found co-overexpression of ETV1 and ETV4 in two cell line models of advanced prostate cancer (MDA-PCa-2b and PC3) and questioned whether these PEA3 family members would cooperate in the acquisition of oncogenic properties or show functional redundancy. Using shRNAs we found that ETV1 and ETV4 have partially overlapping functions, with ETV1 being more relevant for cell invasion and ETV4 for anchorage-independent growth. In vitro expression signatures revealed the regulation of both specific and shared candidate targets that may resemble cellular mechanisms in vivo by interaction with the same intermediate partners. By combining the phenotypic impact data and the gene expression profiles of in vitro models with clinico-pathological features and gene expression profiles of ETS-subtyped tumors, we identified a set of eight genes associated with advanced stage and a set of three genes associated with higher Gleason score, supporting an oncogenic role of ETV1 and ETV4 overexpression and revealing gene sets that may be useful as prognostic markers.

\section{INTRODUCTION}

Chromosomal rearrangements involving different members of the ETS family of transcription factors have been found to occur in prostate carcinomas (PCa) $[1,2]$. The most prevalent gene fusion is the TMPRSS2-ERG, present in nearly half of all human prostate cancers, followed by rearrangements involving the ETV1, ETV4, ETV5 and FLI1 genes, often with promoter fusion partners other than TMPRSS2 [3-10]. These rearrangements, ultimately leading to overexpression of 5' truncated or full-length ETS transcription factors, are considered to be early molecular events in prostate carcinogenesis, not least because they are also found in around $20 \%$ of the pre-cancerous lesion high-grade prostate intraepithelial neoplasia [11]. However, in vivo and in vitro data have shown controversial results regarding the oncogenic role of $E R G$ overexpression, with some studies suggesting that this ETS protein can induce the initiation of neoplastic transformation through the development of pre-invasive lesions [12, 13], whereas others reinforce the need of additional genomic alterations to drive cancer progression $[14,15]$. 
Being part of a large family of 28 members of transcription factors, all sharing the characteristic DNA binding motif GGAA/T [16], it has been questioned whether ETS proteins have redundant or specific functions. Despite the fact that mutual exclusivity of ETS rearrangements is the rule (at least at the cellular level) in prostate carcinomas $[6,17]$ and the impact on cell invasion shared by ERG and ETV1 [12, 18, 19], ETS members show both lack of tissue specificity and co-expression within a tissue [20-22]. Interestingly, our group has recently shown that the ETS transcription factors $E R G$ and ETVI are able to regulate both specific and shared sets of genes in prostate cancer cells [23].

The low frequency of rearrangements involving ETV4, ETV5 and FLI1 (less than 2\%), and the lack of cell line models harboring rearrangements of these ETS members, resulted in scarce knowledge of their oncogenic roles in prostate carcinogenesis. In vitro studies have implicated ETV5 in cell invasion [3, 24-26], alike to $E R G$ and ETV1, while ETV4 seems to be required for both proliferation and anchorage-independent growth [27, 28]. On the other hand, co-expression of PEA 3 family members (ETV1, ETV4 and ETV5) is described to occur in several organs, both during embryonic development and in adult tissues [21, 22]. In 2012, two studies from the same group showed that both ERG and PEA3 rearrangements are found in metastatic lesions, but while ERG rearrangements seem to follow the positiveness seen in the primary tumor, PEA3 rearrangements can occur as clonal events during progression [29, 30]. Moreover, in vivo studies have shown that overexpression of these ETS members can occur during and promote prostate cancer progression [31, 32]. Considering these observations and the high homology between ETV1 and ETV4 (defined by their DNA binding motif), we questioned whether these PEA3 family members would cooperate or have redundant roles in prostate carcinogenesis, by regulating the same or distinct target genes and pathways, and whether in vitro models of ETV1 and ETV4 overexpression could reveal markers of tumor aggressiveness. We used two prostate cancer cell lines (MDA-PCa-2b and PC3) harboring cooverexpression of ETV1 and ETV4 to gain insight into their biological role in vitro, and found both specific and shared candidate target genes that may play a role in tumor aggressiveness in vivo.

\section{RESULTS}

\section{PC3 and MDA-PCa-2b prostate cancer cell lines as in vitro models to study the oncogenic role of ETS co-expression}

Using the TaqMan Low Density Array (TLDA) technology, we evaluated the expression of the five ETS genes known to be involved in genomic rearrangements in PCa (ERG, ETV1, ETV4, ETV5 and FLI1) in a panel of cell lines representing various subtypes of prostate cancer (Figure 1A). Comparing with ETS rearrangement positive $\mathrm{PCa}$, isolated overexpression of $E R G$ was found in $\mathrm{VCaP}$ cells and isolated overexpression of ETV1 was found in LNCaP cells, as reported by others [26]. Interestingly, the prostate carcinoma cell lines PC3 and MDA-PCa-2b exhibited a remarkable coexpression of the three members of the PEA3 subfamily of ETS transcription factors (ETV1, ETV4 and ETV5). FISH analysis was performed in an attempt to find an explanation for the ETV1 and ETV4 outlier expressions in MDA-PCa-2b and PC3 cells. For that purpose, dual color break-apart probes flanking the 5' and 3' regions of each ETS were used. Although no ETV1 rearrangement was found in the PC3 cell line (data not shown), MDA-PCa-2b and LNCaP cells showed chromosomal rearrangements involving the ETV1 locus (7p21.2) (Figure 1B), as described by others [26]. Regarding ETV4, although no chromosomal rearrangement typical of a fusion gene involving this ETS was found, we were able to identify an atypical genomic rearrangement in the PC3 cell line which consisted in the presence of the 5, region of the ETV4 gene located in two distinct marker chromosomes, in addition to three chromosomes 17 with no rearrangements (Figure 1B). No rearrangement was found to mediate the aberrant overexpression of ETV4 in MDA-PCa-2b cells (not shown). Considering the co-overexpression of ETV1 and ETV4 in MDA$\mathrm{PCa}-2 \mathrm{~b}$ and PC 3 cells, we sought to dissect the role of these ETS in prostate carcinogenesis. Two previously established ETV1 models of prostate carcinogenesis [23], namely with silencing and de novo expression of ETV1 (in malignant LNCaP cells and benign PNT2 cells, respectively), were used as complementary cell line models uniquely overexpressing ETV1. We thus used MDA-PCa-2b and PC3 cells to establish two independent ETS downregulated cell populations for each cell line, which stably express shRNAs directed against one target region of ETV1 or ETV4 (explained in the Methods section), along with one negative control (shNeg). Quantitative RT-PCR and immunoblotting analyses showed an efficient decrease of either ETV1 or ETV4 in both MDA-PCa-2b cells (of around 40-60\% and $70-80 \%$, respectively) and PC3 cells (of around $70-80 \%$ and $80-90 \%$, respectively) (Figure 1C), comparing with the shNeg controls. The ETV1 expression levels of the previously established cell line models with significant silencing and de novo expression of ETV1 (LNCaP and PNT2 cells, respectively) was previously shown [23]. The shETV1-LNCaP cells show a decrease of about $90-100 \%$ in ETV1 expression while the PNT2-ETV1 cells used in this study show an increase in ETV1 expression of about $90 \%$ of the expression present in LNCaP cells [23]. 
A

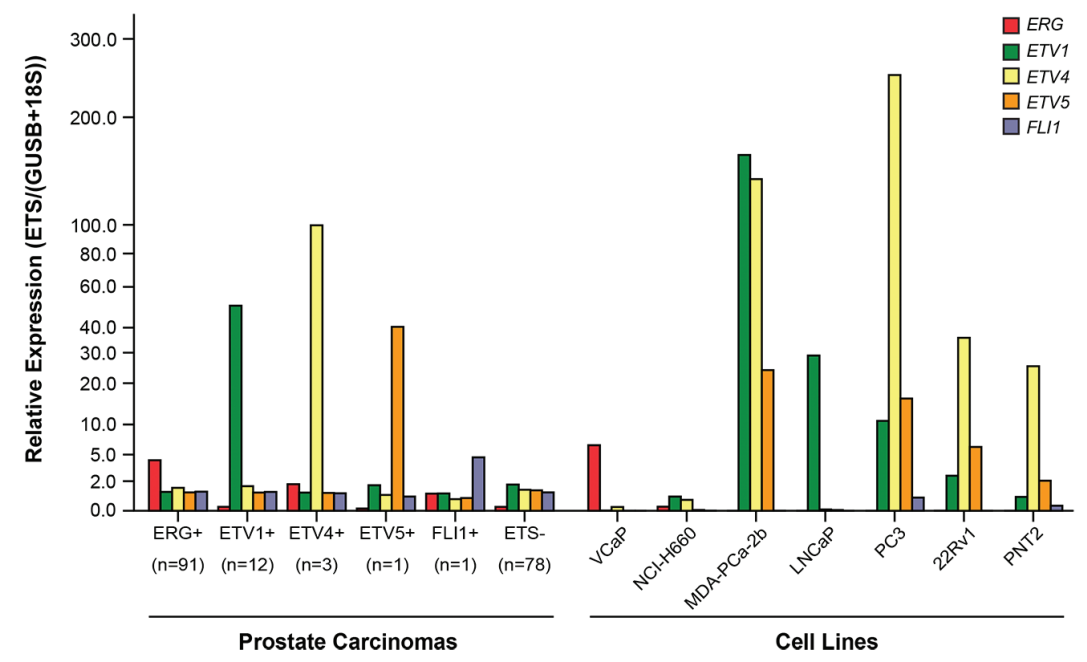

B
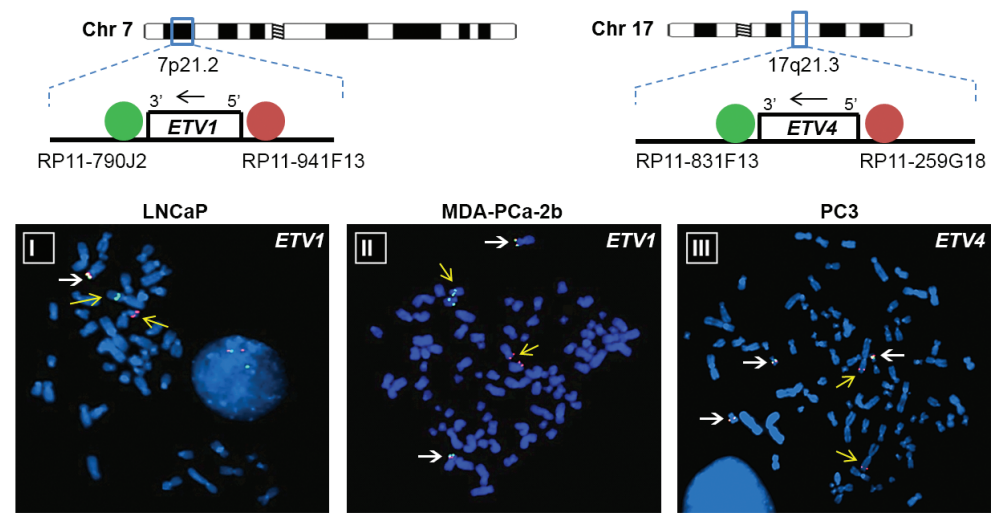

C
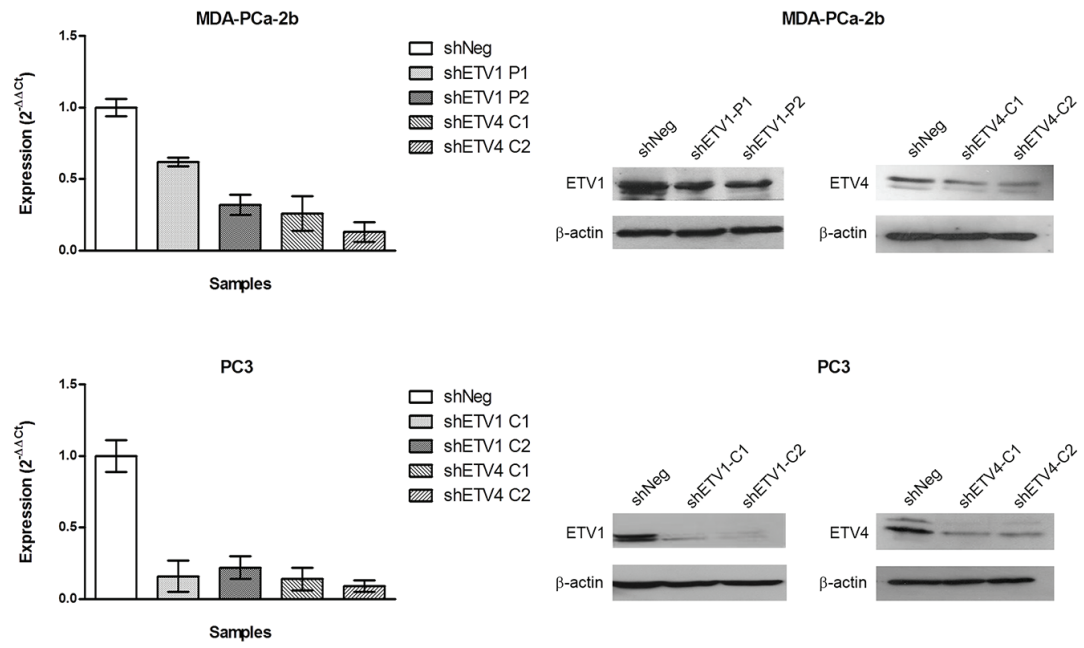

PC3

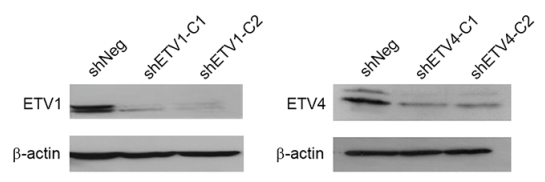

Figure 1: Characterization and establishment of the cell line models. (A) Comparative expression levels of the five ETS transcription factors described to be involved in genomic rearrangements in PCa (ERG, ETV1, ETV4, ETV 5 and FLII) in a panel of prostate carcinomas subtyped for ETS rearrangements and in prostate cell lines by TLDAs. Median-centered values of each ETS were obtained for each sample. For PCa subtypes the average values are shown. (B) FISH analysis for the ETVI locus in LNCaP and MDA-PCa-2b cell lines (I and II, respectively) and for the ETV4 locus in PC3 cells (III). White arrows indicate normal co-localized signals and yellow arrows indicate gene rearrangements. (C) qRT-PCR and immunoblotting for ETV1 and ETV4 after stable silencing of each ETS in the MDA-PCa-2b and PC3 cell lines (upper and lower panels, respectively). A negative control (shNeg) and two independently silenced populations (clonal, $\mathrm{C} 1$ and $\mathrm{C} 2$, or polyclonal, $\mathrm{P} 1$ and $\mathrm{P} 2$ ) for the same target region were analyzed. 


\section{Silencing of either ETV1 or ETV4 does not impact proliferation or apoptosis in a co-expression background}

To evaluate the possible effect of sustained ETVI or ETV4 knockdown on PCa cell growth and apoptosis in vitro we used the MTT and the APOPercentage ${ }^{\mathrm{TM}}$ assays, respectively. Silencing of ETV1 or ETV4 did not alter the growth rate or apoptosis levels in both ETS co-expressing models (MDA-PCa-2b and PC3) (Figure 2). Although a slight increase in apoptosis was observed, results do not reach statistical significance. In LNCaP cells, which only express ETV1, there is a clear tendency for higher apoptotic levels in the ETV1 silenced populations, although statistical analysis show lack of significance $(p>0.05)$.

\section{ETV4 overcomes ETV1 in the regulation of anchorage-independent growth in a co-expression cellular context}

To determine the involvement of ETV1 and ETV4 in the ability of cells to grow in the absence of an anchorage surface, associated with the metastasis process, we used an in vitro soft-agar assay. In contrast to shNeg cells, shETV4 cells showed a significant decrease in colony formation after two weeks in culture, originating $20 \%$ fewer colonies in MDA-PCa-2b cells and around $50-60 \%$ in PC3 cells (Figures 3A and 3B). No differences were observed in ETV1 silenced cells from both cell line models. Contrarily, stable silencing of ETV1 in LNCaP cells resulted in a highly significant decrease of more than
A

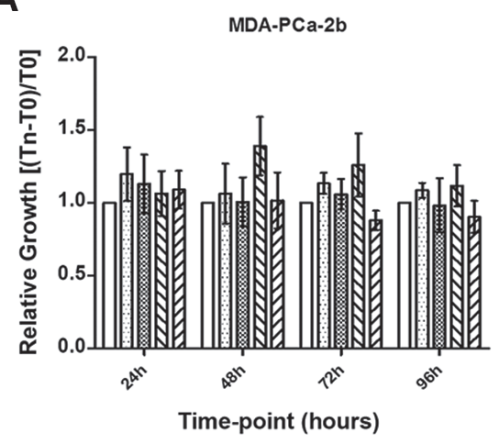

B

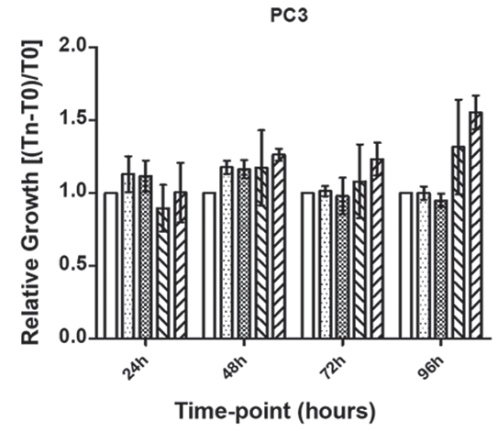

C

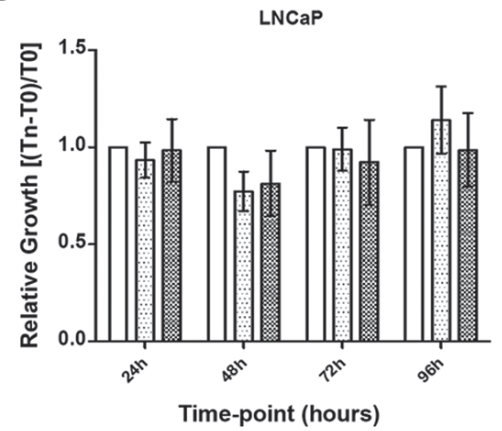

$\square$ shNeg ShETV1P1 shETV1P2 ShETV4 C1 ED ShETV4 C2

$\square$ shNeg ShETV1 C1 ShETV1 C2 ShETV4 C1 ED ShETV4 C2

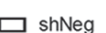
四 ShETV1 C1 ShETV1 C2
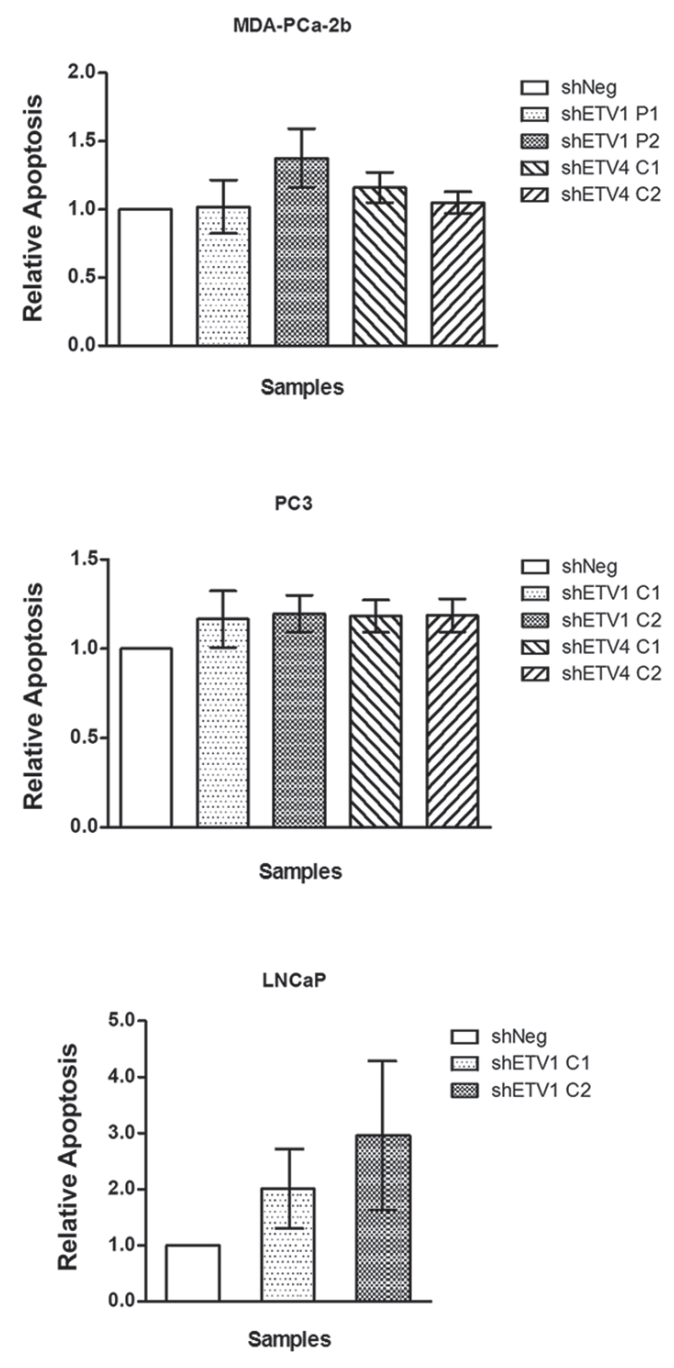

Figure 2: In vitro evaluation of the impact of ETV1 or ETV4 silencing in proliferation and apoptosis. (A-C) Relative growth was estimated for four time-points in culture (left) and apoptosis for one time-point ( $96 \mathrm{hr}$, right), for the three cell line models with manipulated ETV1 or ETV4 expression: MDA-PCa2b (A), PC3 (B) and LNCaP (C) cell lines. Results are shown for each silenced cell population relative to the shNeg cells from three independent experiments. There are no statistically significant results $(p>0.05)$. 
A
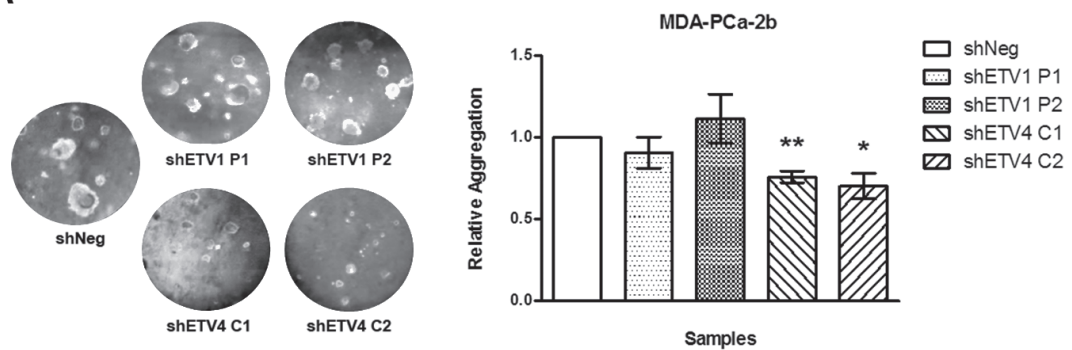

B
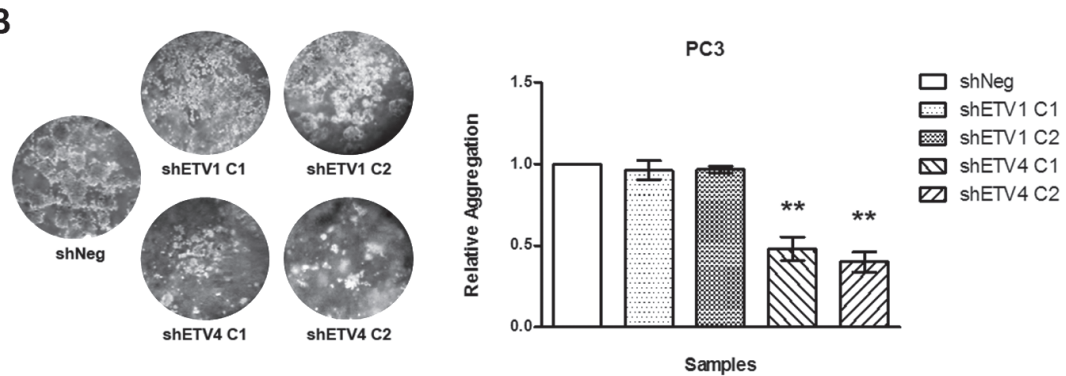

C

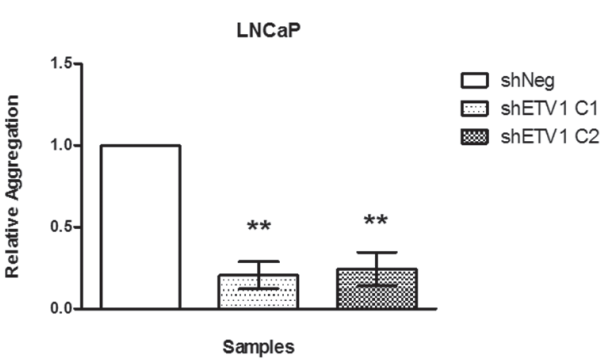

D
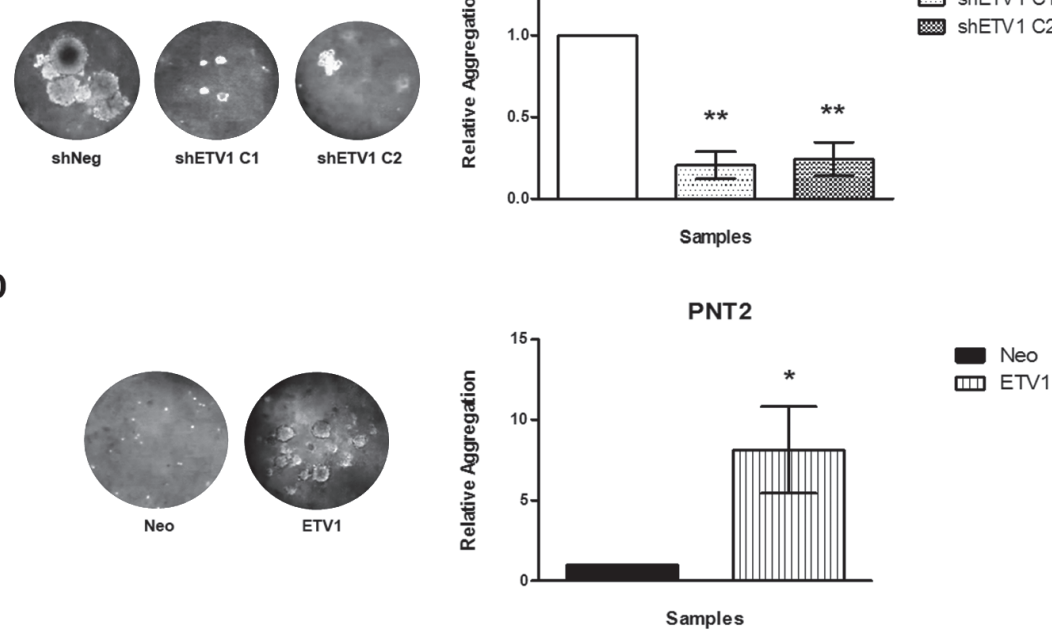

Figure 3: In vitro evaluation of the impact of ETV1 or ETV4 silencing in anchorage-independent growth. (A-D) Qualitative visualization (left $)$ and quantitative analysis $(r i g h t)$ of the several cell line models with manipulated ETV1 or ETV4 expression: MDA-PCa-2b (A), PC3 (B), LNCaP (C), and PNT2 (D) cell lines. Results are shown for each silenced cell population relative to the shNeg cells from three independent experiments. Statistically significant $p$ values are showed by an asterisk $\left({ }^{*} p<0.05 ; * * p<0.01\right)$.

$70 \%$ in the number of colonies formed (Figure 3C). An inverse effect was observed in PNT2 cells with de novo ETV1 overexpression, showing nearly ten-fold more colonies than the respective control (Figure 3D).

\section{Knockdown of ETV1 inhibits cell invasion in vitro with a greater impact than knockdown of ETV4}

We conducted in vitro invasion assays to evaluate the role of ETV1 or ETV4 in the ability of MDA-PCa2b, PC3, LNCaP and PNT2 cells to invade through a basement membrane matrix. MDA-PCa-2b-shNeg cells revealed low invasive capacity, and knockdown of either ETV1 or ETV4 did not change that characteristic (Figure 4A). On the other hand, a significant decrease in cell invasion was observed in both shETV1 and shETV4 cells derived from the PC3 cell line, with a much stronger decrease seen in shETV1 cells $(80-90 \%$ decrease in shETV1 cells versus $40-50 \%$ decrease in shETV4 cells, Figure 4B). In LNCaP cells, silencing of ETV1 expression led to a significant $50-60 \%$ decrease in invasion when compared to the shNeg control (Figure $4 \mathrm{C}$ ), whereas in PNT2 cells, de novo overexpression of ETV1 induced an inverse effect, leading to a ten-fold increase in cell invasion when compared to the PNT2Neo control cells (Figure 4D). 
A
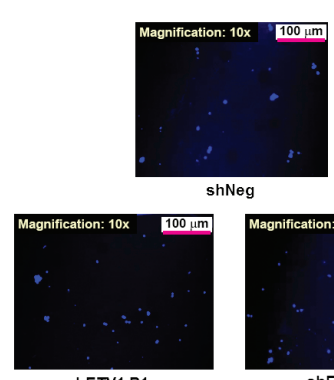

ShETV1 P1
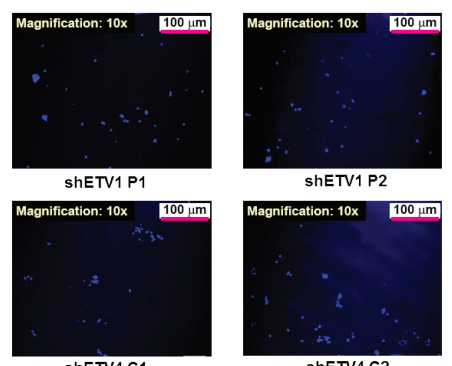

ShETV4 C1

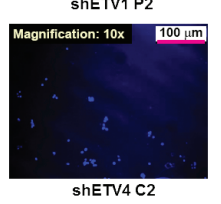

B
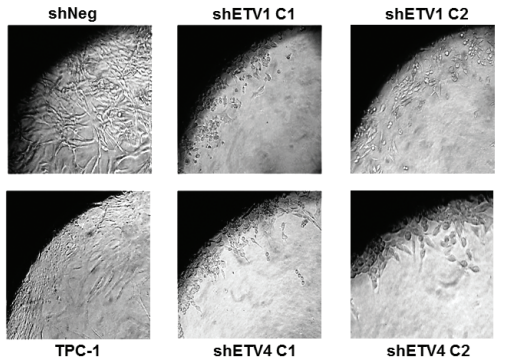

C
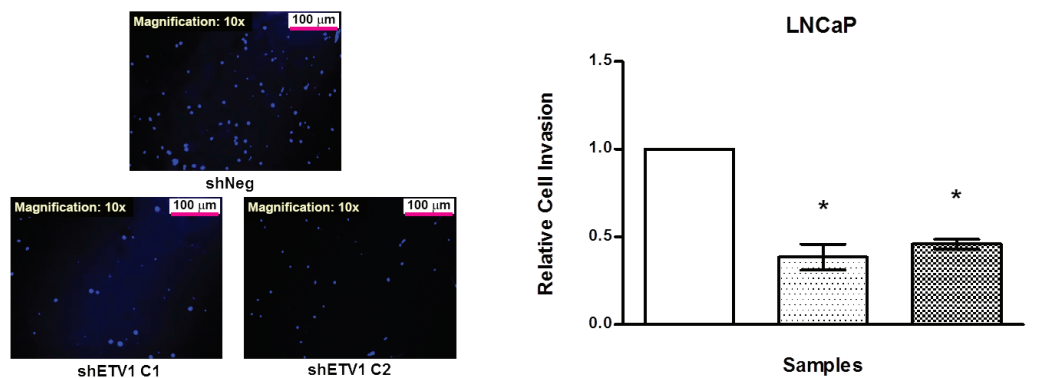

D

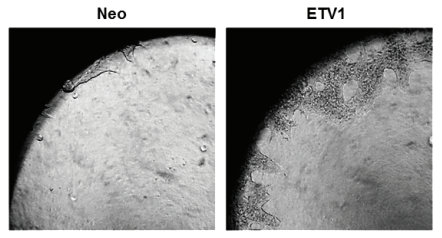

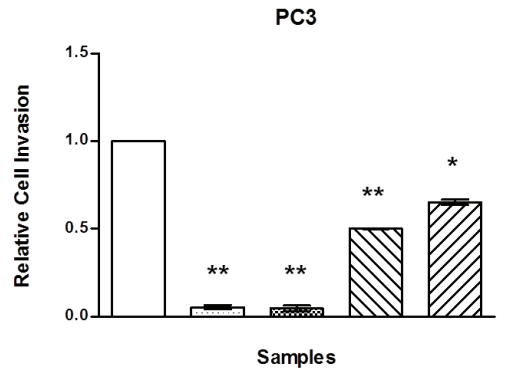
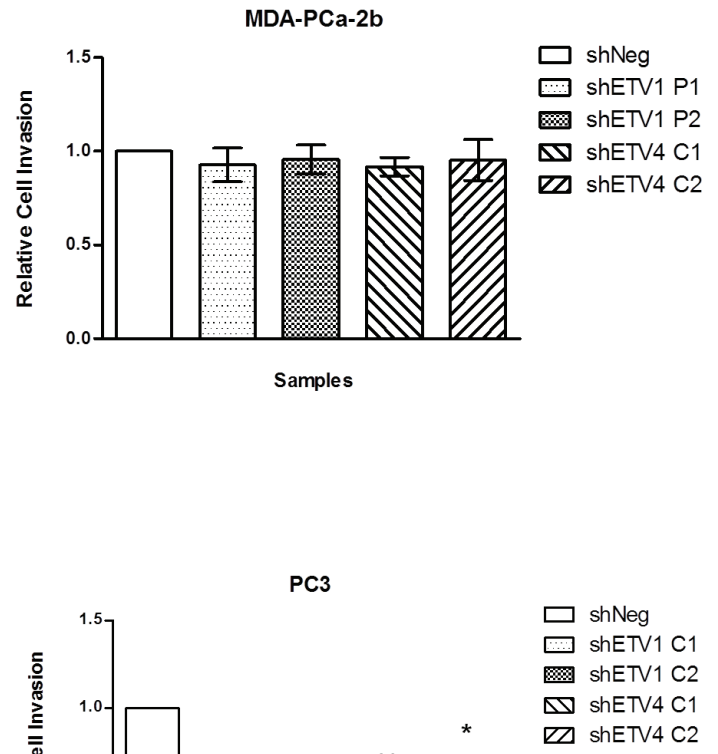

$\square$ shNeg

shETV1 C1

shETV1C2

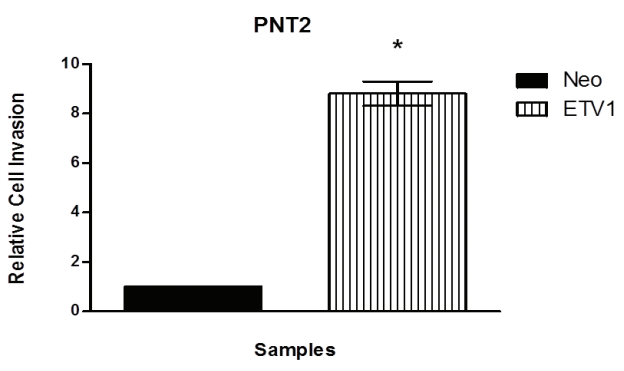

Figure 4: In vitro evaluation of the impact of ETV1 or ETV4 silencing in cell invasion. (A-D) Qualitative visualization (left) and quantitative analysis (right) of the several cell line models with manipulated ETV1 or ETV4 expression: MDA-PCa-2b (A) PC3 (B), LNCaP (C) and PNT2 (D) cells. Results are shown for each silenced cell population relative to the shNeg cells from three independent experiments. Statistically significant $p$ values are showed by an asterisk $(* p<0.05 ; * * p<0.01)$. 
A

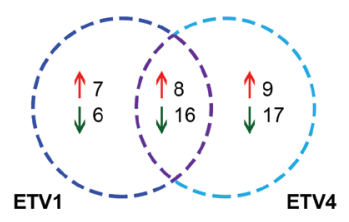

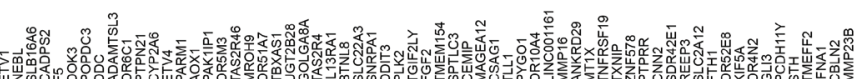

$\stackrel{0}{+}$
$\stackrel{-}{-}$
$\stackrel{9}{+}$
MDA-PCa-2b-ETV4

PC3-ETV4

MDA-PCa-2b-ETV1

LNCaP-ETV1

PNT2-ETV1
B

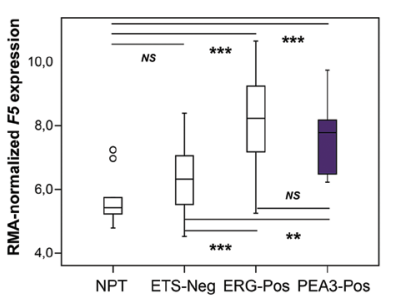

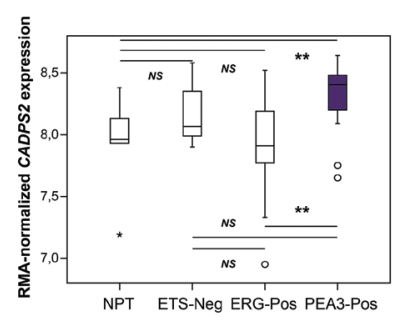

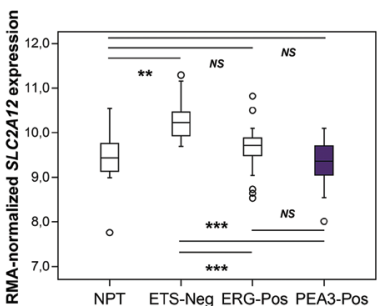

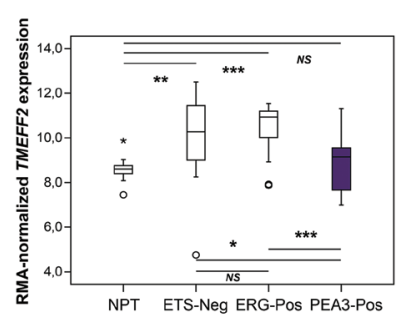

C

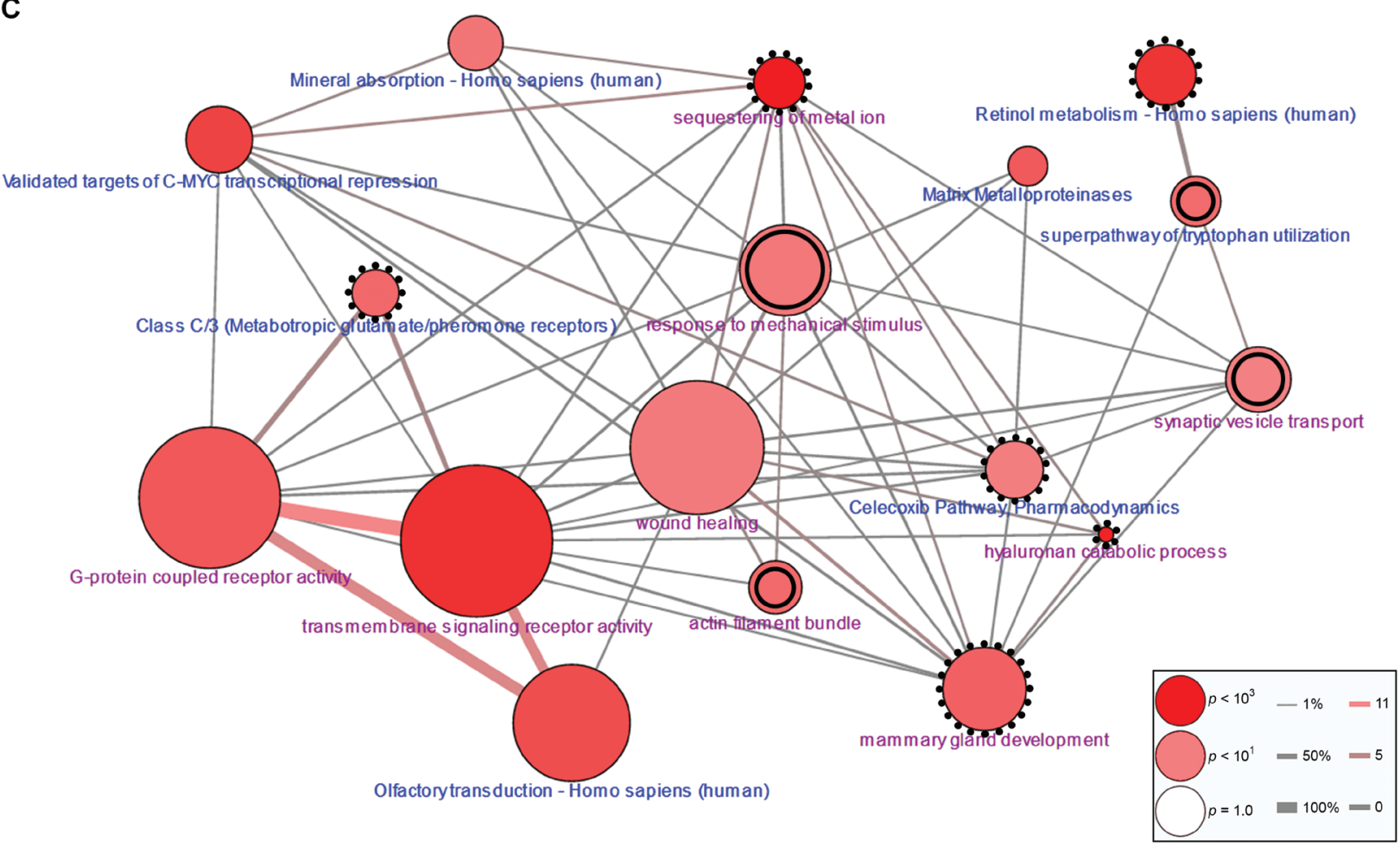

Figure 5: Dissection of ETV1 and/or ETV4 regulated genes. (A) Venn-diagram (left) and hierarchical clustering (right) of the 61 ETV1 and/or ETV4 associated genes in vitro, showing specific and shared candidate targets (Supplementary Table 1). ETV1 and ETV4 are also included. (B) Box-plot distribution of the expression of the four ETV1 and/or ETV4 associated genes in vitro that discriminate PEA3 positive prostate carcinomas from other tumors or NPT samples. NS - not significant, $(* p<0.05 ; * * p<0.01 ; * * *<0.001)$. (C) Gene set enrichment network of Gene Ontology Categories (in pink) and Molecular Pathways (in blue) for the panel of 61 ETV1 and/or ETV4 associated genes in vitro (Supplementary Table 2). Specific enrichment of ETV1 target genes is shown in double-lined circles, specific enrichment of ETV4 target genes in dotted circles and enrichment of both ETV1 and ETV4 targets in simple-lined circles. Connector line thickness represents the percentage of genes shared between categories and line color-code represents the number of shared genes in the input list. Circle size reflects the number of genes in the category and color-grading represents $p$ value of enrichment in the input list. 


\section{Whole transcriptome expression profile of in vitro cell models reveals candidate PEA3 targets in rearrangement-positive tumors}

We generated an integrated transcriptomic analysis to gain mechanistic insights into the effect of ETVI and ETV4 in prostate carcinogenesis, and to identify putative target genes. We identified 61 genes that were differentially expressed upon ETV1 or ETV4 knockdown (fold-change higher than 1.5 or lower than -1.5 ) (Figure 5A). A set of genes was found associated with ETVI overexpression, with seven genes showing increased expression and six genes showing decreased expression (Supplementary Table 1). On the other hand, the expression of nine and 17 genes was found to be positively and negatively associated with ETV4 overexpression, respectively (Supplementary Table 1). These sets included two genes (TLL1 and PYGO1) that were present in both ETV1- and ETV4-specific putative target genes, but their expression was inversely regulated by these ETS members, being upregulated by ETV1 and down-regulated by ETV4. A set of genes was associated with both ETS when considering that the same fold-change was present in three of the four cell line models, which included eight up-regulated genes and sixteen down-regulated genes (Supplementary Table 1). To evaluate whether any of these genes was differentially expressed in $\mathrm{PCa}$ harboring PEA3 rearrangements, we used our data from a series of $50 \mathrm{PCa}$ and nine NPT samples [23]. We found four genes that show association with PCa harboring PEA3 rearrangements (Figure 5B). F5 and $S L C 2 A 12$ can differentiate PEA3-positive from ETSnegative tumors ( $p<0.01$ and $p<0.001$, respectively) while CADPS2 and TMEFF2 can differentiate PEA3positive from ERG-positive tumors $(p<0.01$ and $p<0.001$, respectively).

\section{Gene set enrichment analysis shows enrichment of PEA3 specific and shared GO categories of genes}

The functional gene ontology (GO) evaluation considered around $95 \%$ and $90 \%$ of the genes in the ETV1 and ETV4 gene sets, respectively, to be involved in at least one GO category. On the other hand, $63 \%$ of the genes (both of ETV1 and ETV4 genes sets) are described to be present in at least one pathway. The major enrichment (both in GO annotation and pathway analysis, Supplementary Table 2) involves genes that codify membrane receptors associated with olfactory compounds, with three genes being deregulated by both ETS transcriptions factors (OR10A4, OR52E8, OR4N2) and one (OR6C1) and two (OR5M3 and OR51A7) genes of the same family being specifically deregulated by ETV1 and ETV4, respectively (Figure 5C). Other PEA3shared enriched pathways include the deregulation of matrix metalloproteinases (MMP23B and MMP16) and of validated targets of C-MYC transcriptional repression
(FTH1 and TMEFF2). On the other hand, ETS-related pathway analysis revealed PEA3-specific deregulation of metabolic pathways, namely the deregulation of the tryptophan degradation pathway by ETV1 and the deregulation of the retinol metabolism pathway by ETV4 (Supplementary Table 2). Other PEA3-specific gene ontology categories include the ETV4-specific regulation of the expression of genes associated with sequestering of metal ion (DDIT3 and FGF2) and of two additional members (as the olfactory receptors mentioned before) of the family of G-protein coupled receptors (GPCR) associated with taste recognition (TAS2R4 and TAS2R46) and classified as metabotropic glutamate/pheromone receptors.

\section{Interaction network analysis reveals ETS specific key players of identical signaling networks}

Using the full list of 61 genes associated with ETV1 and ETV4 in vitro in the "induced network module" tool of the ConsensusPathDB database, seven genes were not mapped to any protein, one ETV1-specific (POPDC3), two ETV4-specific (MROH9, PARM1) and four shared by both ETS (ANKRD29, LINC00161, PCDH11Y, STH). When the remaining 54 PEA3-associated target genes were analyzed together with ETV1 and ETV4, five ETV1-specific (CYP2A6, DDC, F5, NEBL and PTPN21), seven ETV4-specific (AOX1, DDIT3, FGF2, PAK1IP1, PLK2, SLC22A3 and TGIF2LY) and seven PEA3-shared (CNN2, FTH1, GLI3, IFNA1, MT1X, PTPRR and TXNIP) targets showed known protein or biochemical interactions by shared intermediate partners (Figure 6A). When we crossed the ETV1 gene set here identified $(n=$ 38) with our previously identified list of tumor-associated ETV1 target genes $(n=43)$ no gene overlap was seen (Supplementary Table 3), but ten (26.3\%) of the in vitroassociated ETV1 candidate target genes and 17 (39.5\%) of the tumor-associated ETV1 candidate target genes showed interaction with shared intermediate partners (Figure 6B).

\section{Combined evaluation of the phenotypic impact and gene expression profile reveals candidate players of invasion and anchorage-independent growth associated with clinico-pathological characteristics of tumor aggressiveness}

Considering the absence of an overlap between ETV1 target genes in vitro and in vivo, we questioned whether we could use the observed impact of ETV1 and ETV4 silencing in invasion and AIG to find phenotypeassociated genes that could be useful as markers of tumor aggressiveness in vivo. Crossing the gene expression profiles of the cell line models showing impact in invasion and/or AIG (Figure 7A) we defined a set of 81 genes with potential to be involved in these oncogenic 
A

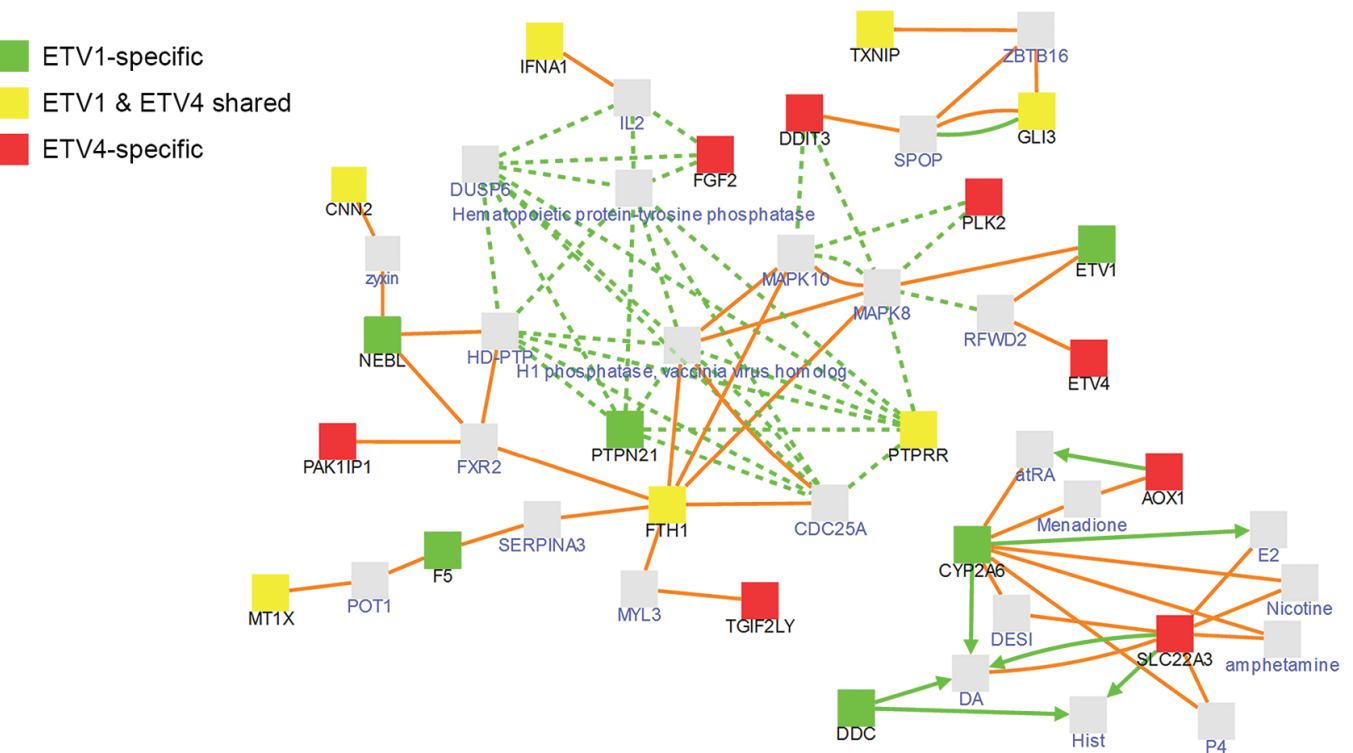

B

Tumor-associated ETV1 candidate target genes

In vitro-associated ETV1 candidate target genes

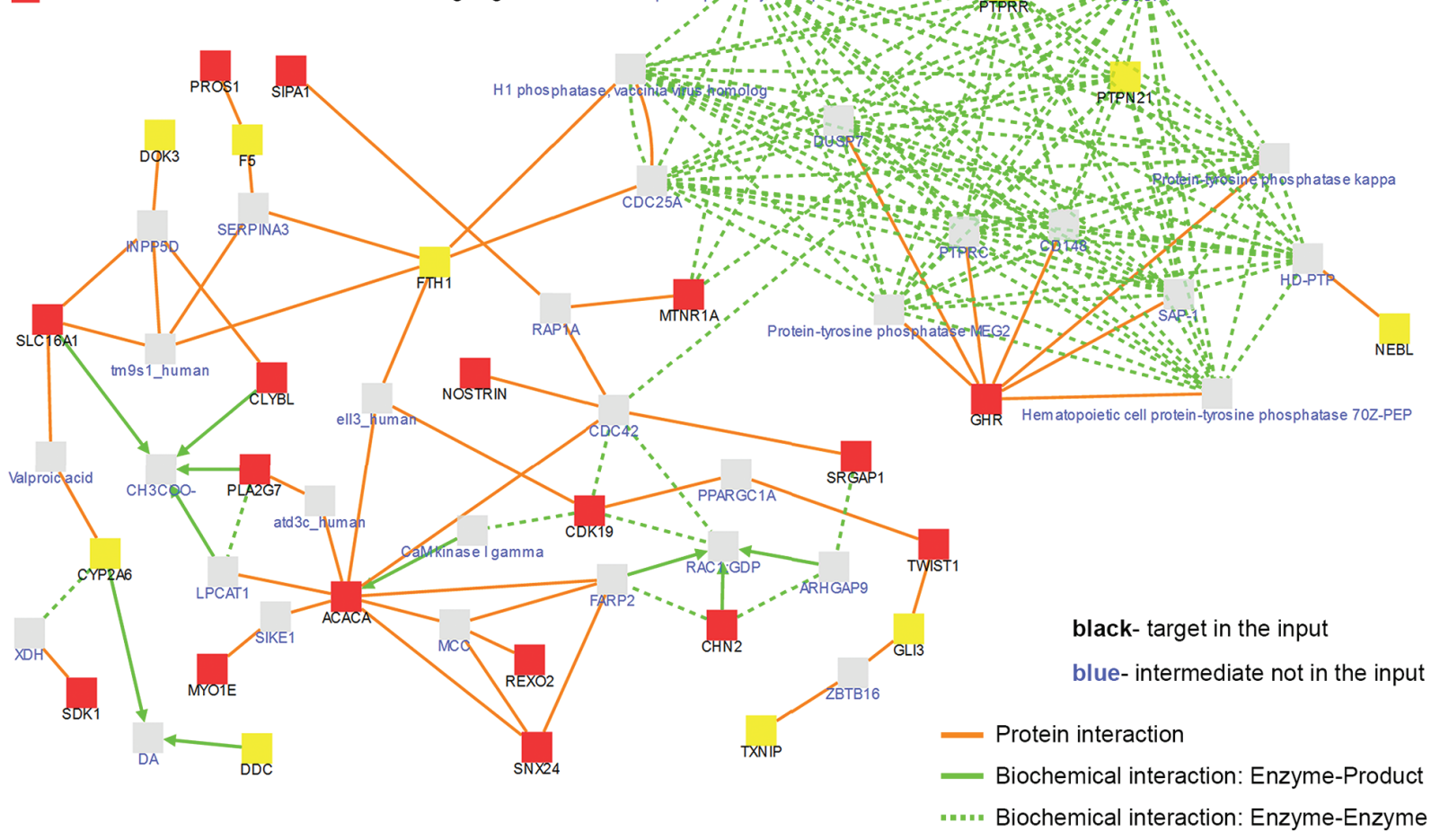

Figure 6: Dissection of ETV1 and/or ETV4 interaction networks. (A) Interaction networks of ETV1 and/or ETV4 associated genes in vitro and known intermediate partners (Supplementary Table 1). (B) Interaction networks of in vitro and in vivo ETV1 associated genes and known intermediate partners (Supplementary Table 3). Proteins from the input list are named in black with color-coded squares and known intermediates not in the input list are named in blue with grey squares. Connector lines are color-coded for the type of interaction: protein interactions in orange and biochemical interactions in green.

properties (Supplementary Table 4). A set of 17 genes was considered to be more associated with AIG and a set of 27 genes with invasion. Looking for clinicopathological associations using the expression profile of our series of 50 prostate carcinomas [23], we found two genes (ATF3 and GSTM4) significantly decreased ( $p=0.006$ and $p=0.015$, respectively) and one gene (OR5M3) significantly increased $(p=0.020)$ in prostate 
A
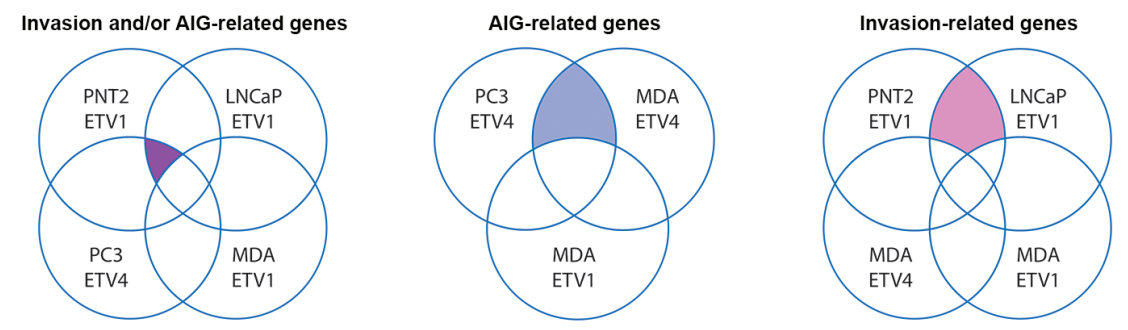

B
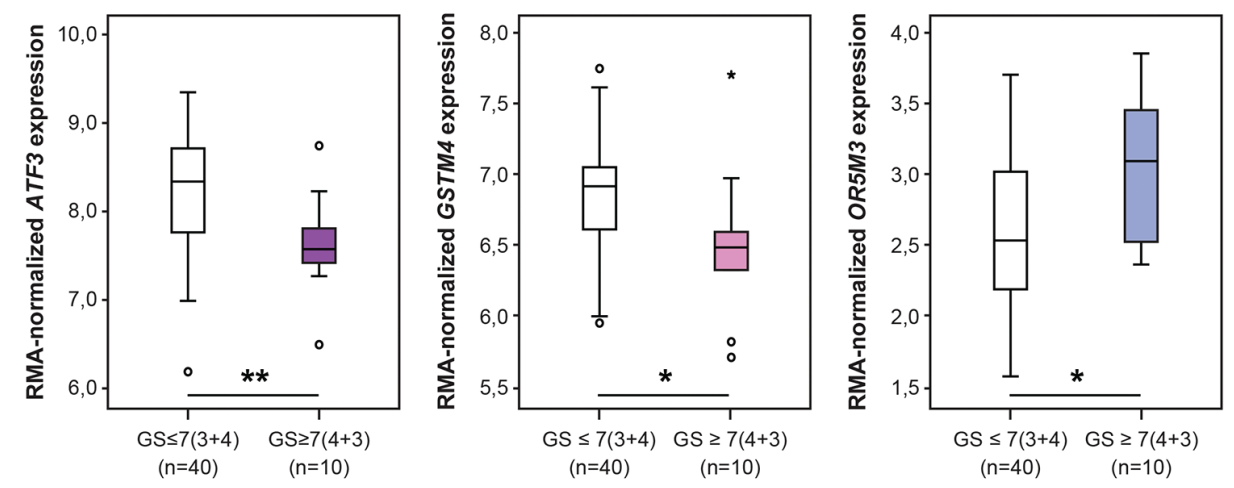

C
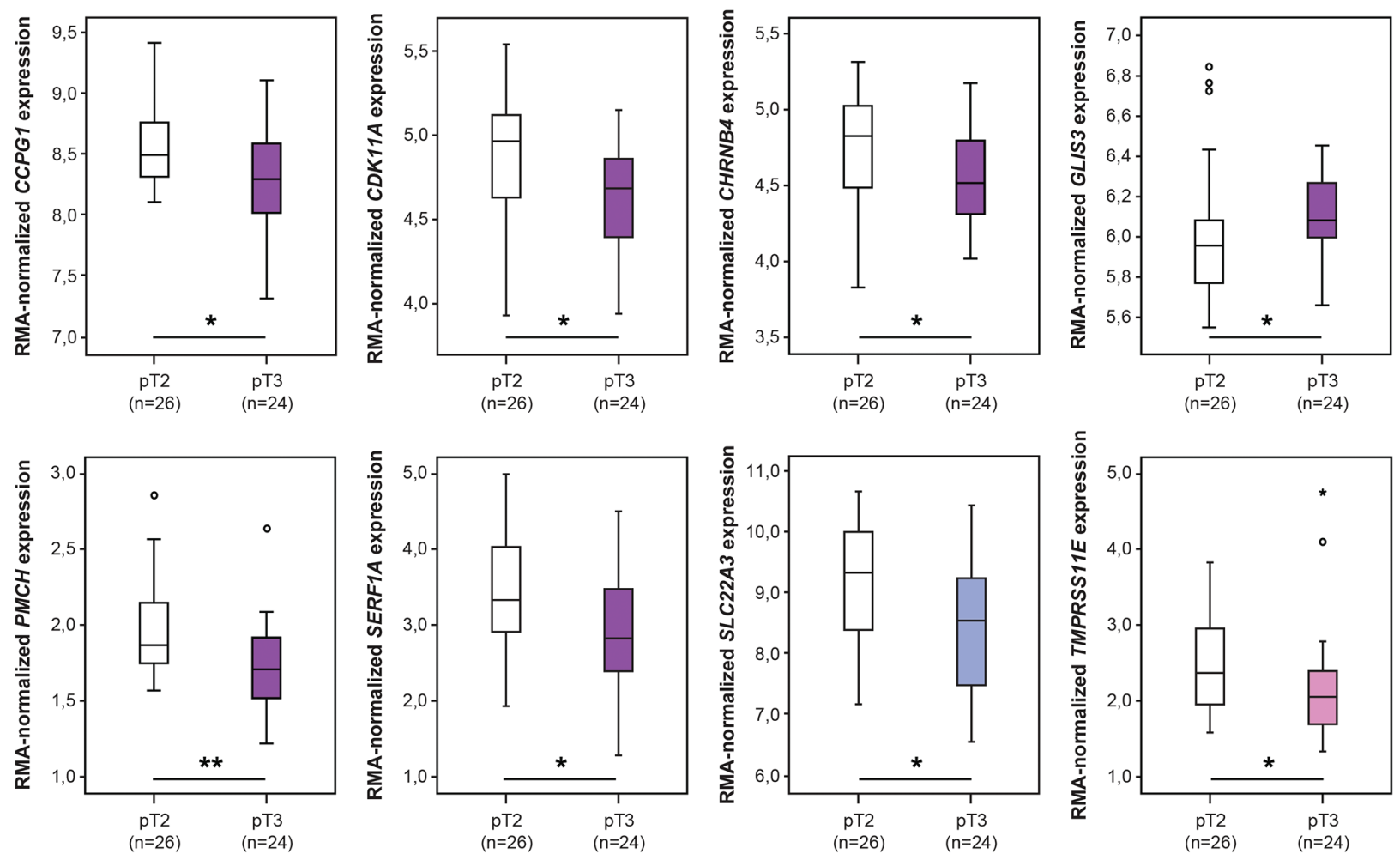

Figure 7: Association between the expression of invasion and/or AIG-related genes in vitro and prognostic factors in vivo. (A) Schematic representation of the cell line models used to define invasion and/or AIG-related genes in vitro. Left, venn-diagram of genes associated with invasion and/or AIG; center, venn-diagram of genes associated with AIG; right, venn-diagram of genes associated with invasion. (B) Box-plot distribution of the expression of the three invasion and/or AIG-related genes associated with higher Gleason score $(\mathrm{GS}=7(4+3))$. (C) Box-plot distribution of the expression of the eight invasion and/or AIG-related genes associated with invasive tumors (pT3). The color of the box-plots refers to the venn-diagram from where each gene was obtained. Statistically significant $p$ values are showed by an asterisk between box-plots $\left({ }^{*} p<0.05 ;{ }^{* *} p<0.01\right)$. 
Table 1: Performance of the panel of seven genes as biomarkers for the identification of tumors with local invasion using different cut-off values

\begin{tabular}{|c|c|c|c|c|c|}
\hline Cut-off (genes in cut-off) & $p$ value & PPV & NPV & Sensitivity & Specificity \\
\hline Percentil 10 (1 gene) & 0.00017 & $77 \%$ & $76 \%$ & $71 \%$ & $81 \%$ \\
\hline Percentil 25 ( 2 genes) & 0.00005 & $76 \%$ & $81 \%$ & $79 \%$ & $78 \%$ \\
\hline Percentil 50 (4 genes) & 0.00292 & $67 \%$ & $75 \%$ & $75 \%$ & $67 \%$ \\
\hline
\end{tabular}

carcinomas with higher Gleason score $(\mathrm{GS} \geq 7(4+3))$ relative to prostate carcinomas with lower Gleason score $(\mathrm{GS} \leq 7(3+4))$ (Figure 7B). On the other hand, seven genes (CCPG1, CDK11A, CHRNB4, PMCH, SERF1A, $S L C 22 A 3$ and TMPRSS11E) were found significantly decreased $(p=0.046, p=0.012, p=0.016, p=0.006$, $p=0.044, p=0.021$ and $p=0.040$, respectively) and one gene $(G L I S 3)$ significantly increased $(p=0.042)$ in pT3 invasive tumors relative to organ confined pT2 tumors (Figure 7C). All these associations were independent of age at diagnosis and PSA at diagnosis. We further explored the clinical utility of this panel of genes in predicting locally invasive tumors combining different expression cut-offs (Table 1). Tumors with decreased expression (bottom 25\%) of at least two of these genes (CCPG1, CDK11A, CHRNB4, PMCH, SERF 1A, SLC22A3 and TMPRSS11E) show the strongest correlation with local invasion $(p=0.00005)$. GLIS3 was excluded from the gene panel for its overlapping distribution in pT2 and pT3 tumors.

\section{DISCUSSION}

Overexpression of oncogenic ETS transcription factors has been associated with chromosomal rearrangements that result in the formation of gene fusions in $50-70 \%$ of the prostate cancers $[2,33,34]$. However, information about the biological role of these ETS transcription factors and their downstream target genes remains insufficient. Screening a panel of prostate cancer cell lines for the expression of the five ETS genes described to be involved in genomic rearrangements in PCa (ERG, ETV1, ETV4, ETV5 and FLI1), we found two cell lines - MDA-PCa-2b and PC3 - showing co-overexpression of ETV1 and ETV4. In fact, a similar profile was reported by Hollenhorst et al. for PC3 cells [27], but ETV1 was not considered as overexpressed and the MDA-PCa-2b cell line was not evaluated. Using the expression data of our series of prostate carcinomas subtyped for ETS rearrangements [6], we show that both PC 3 and MDA-PCa-2b cells have co-overexpression of ETV1 and ETV4, both ETS in higher levels than those of $E R G$ in $\mathrm{VCaP}$ cells, the in vitro model of the TMPRSS2$E R G$ rearrangement [12]. MDA-PCa-2b and $\mathrm{PC} 3$ cells were thus used as suitable models to investigate whether ETV1 and ETV4 have similar mechanistic and functional involvement in tumor biology. A recent study selected the
DU-145 prostate cancer cell line as a model to address the oncogenic role of ETV4 in prostate cells [28]. Although this cell line was not included in our initial panel, it could be a valuable model to study the individual role of ETV4 overexpression in prostate carcinogenesis, which led us to acquire DU-145 from DSMZ and perform qRTPCR and western blot for both ETS. Our data, however, revealed no ETV4 (or ETV1) overexpression at mRNA or protein levels in DU-145 (not shown), eventually reflecting differences in the DU-145 cell lines studied between the two groups. Looking for the presence of chromosomal rearrangements that might have occurred within the ETV1 and ETV4 genes in PC3 and MDA$\mathrm{PCa}-2 \mathrm{~b}$ cells, and contrarily to what was reported by Hollenhorst et al. [27], we were able to identify a structural genomic alteration in the PC3 cell line that consists in the presence of the 5' region of the ETV4 gene in two similar aberrant chromosomes, in addition to three chromosomes 17 with no rearrangement. Bearing in mind the negative nature of PC3 cells for androgen receptor expression [35], androgens are excluded as possible mediators of ETV4 overexpression, and the regulatory mechanism remains elusive. As for the MDA-PCa- $2 b$ cells, no genomic rearrangement was found to mediate the aberrant overexpression of ETV4. Consistent with earlier work [26], an ETV1 rearrangement was found in the MDA-PCa-2b cell line, validating its authenticity and the cause of the ETV1 outlier expression. Considering ETS rearrangements as primary events, it is possible that in $\mathrm{PC} 3$ cells the structural rearrangement of ETV4 preceded altered ETV1 expression, while in MDA-PCa$2 \mathrm{~b}$ cells the ETV1 rearrangement might have occurred before ETV4 overexpression.

In order to gain insight into the phenotypic role of each ETS for prostate carcinogenesis, several in vitro experiments were performed. When evaluating the effects of ETV1 and ETV4 silencing in AIG by the colony-forming assay, we observed that ETV4 knockdown strongly impaired the ability of PC3 and MDA-PCa-2b cells to grow in an anchorageindependent way, significantly decreasing the number of colonies formed. These results match those reported by Hollenhorst et al. in PC3 cells and are consistent with data reported by Pellecchia et al. in DU-145 cells [27, 28], suggesting that high ETV4 expression levels are necessary for robust AIG. Surprisingly, loss of this property was not observed after depletion of ETV1 in the same cell line models, contrasting with the phenotype observed after knockdown of 
ETV1 in the LNCaP cell line and after de novo expression of ETV1 in the benign PNT2 cells (in line with other reports $[25,36])$. Altogether, it is thus reasonable to hypothesize that downregulation of ETV1, although not sufficient to revert the cells' ability to form colonies in a co-expression context, appears to be a critical factor when present as a uniquely overexpressed ETS, presumably reflecting an overlapping function with ETV4. This hypothesis could also explain the observed impact in apoptosis of ETVI silencing in the $\mathrm{LNCaP}$ cell line (although not reaching statistical significance), while no impact was observed in the co-expressing cell line models. However, and although we did not find a cell line model uniquely overexpressing ETV4, Pellecchia et al. (2012) reported no impact in apoptosis after ETV4 silencing in their DU-145 cells [28], suggesting that ETV1 involvement in apoptosis may be specific of LNCaP cells, instead of being masked by an ETV4 overlapping function in MDA-PCa$2 \mathrm{~b}$ and $\mathrm{PC} 3$ cells. Studies in other tumor types also support an involvement of ETV1 and ETV4 in AIG [37, 38], but no reference was made regarding the comparative expression of these ETS in the cell models used. Our data, however, point to a stronger involvement of ETV1 in the acquisition of invasive properties. In fact, although a significant decrease of cell invasion was observed after knockdown of either ETV1 or ETV4 in PC3 cells, the global effect of ETV1 knockdown in cell invasion was much more dramatic than the effect of ETV4 knockdown in this cell line model. Moreover, a significant gain of cell invasion was obtained by de novo overexpression of ETV1 in the benign PNT2 cells, while a significant decrease was observed after ETV1 knockdown in the malignant $\mathrm{LNCaP}$ cells. These results are consistent with the invasive growth promoting effect described for ETVI and $E R G$ by other groups $[12,24]$. The lack of impact of silencing of either ETV1 or ETV4 in the invasive properties of the MDA-PCa-2b cell line may either reflect overlapping functions of these ETS or the existence of other critical factors that promote an invasive phenotype in this cellular context. Consistent with this, Pellecchia et al. (2012) reported that, while silencing of ETV4 in DU-145 cells did not impair cell invasion, de novo expression in the nonmalignant RWPE cells lead to a gain of this oncogenic characteristic [28].

Aiming to find molecular players of either ETV1 or ETV4 regulation, we analyzed expression changes between the in vitro manipulated cell populations and their respective controls using whole transcriptome exon-level expression arrays. As it has been reported for other ETS transcription factors, and in line with what we have observed concerning the phenotypic impact of ETV1 and ETV4, we found that these members of the PEA3 subfamily of ETS transcription factors are involved in the regulation of both specific and shared molecular partners/pathways. The most surprising data from the gene set enrichment analyses is the deregulation of six members of the family of olfactory receptors, either PEA3 specific or PEA3 shared. Moreover, when searching for phenotype-associated gene expression changes three additional member of this family were found (Supplementary Table 4) and one, OR5M3, showed to be associated with higher Gleason score, with potential to be used as a biomarker. The involvement of this type of receptors in cancer development in general has been scarcely explored. In fact, one of the first reports comes from $\mathrm{Xu}$ and collaborators with the description of the overexpression of OR51E2, first called PSGR (prostatespecific G-protein-coupled receptor), in around $60 \%$ of the prostate carcinomas [39], the same gene that we have recently found to be a 5' fusion partner of ETV1 [7]. In 2009, Neuhaus and collaborators reported beta-ionone as an activator of OR51E2, suggesting the potential usefulness of specific receptor ligands as therapeutic approaches [40]. Interestingly, the ETV4-specific gene set also revealed deregulation of two taste receptors, members of a largely unexplored subfamily of $\mathrm{G}$ proteincoupled receptors (GPCR) that together with olfactory receptors account for over half the GPCR repertoire [41]. As GPCR are cell surface receptors and can be activated by a plethora of stimuli, from photons to peptides, hormones and lipids [41], studies focusing in the search for specific ligands of these olfactory and taste receptors could reveal potential therapeutic approaches for tumors harboring overexpression of these PEA3 members [42]. Other PEA3 deregulated genes include the "deregulation of matrix metalloproteinases" and of "validated targets of C-MYC transcriptional repression", two GO terms familiar to ETS involvement in prostate carcinogenesis. Despite the in vitro association between the expression of both ETVI and ETV4 and the expression of MMP16 and $M M P 23 B$, we found no correlation in prostate carcinomas harboring PEA3 rearrangements, suggesting a cell type and microenvironment specific regulation of MMPs [43]. Of the genes included in the GO category of "validated targets of C-MYC transcriptional repression", $T M E F F 2$ is worth to be explored. TMEFF2 codifies an androgen-regulated transmembrane protein, normally restricted to the brain and prostate tissues, initially described as a tumor suppressor gene for its inhibition of cell growth and hypermethylated status in several tumors, including prostate carcinomas $[44,45]$. Conversely, other reports showed that a significant proportion of prostate carcinomas exhibit TMEFF2 overexpression [46, 47] and in vivo studies using LNCaP xenografts show that TMEFF2 inhibition results in tumor growth arrest [46], favoring its role as an oncogene. While this oncogenic prostate-specific role of TMEFF2 is in line with our observed positive association between TMEFF2 and $E T V 1$ and ETV4 expression, being significantly decreased with silencing of either ETS, our panel of PCa subtyped for ETS rearrangements [23] showed an association of $T M E F F 2$ overexpression with non-PEA3-positive PCa. This dual activity can result from different $T M E F F 2$ isoforms, produced from either alternative splicing or cleavage of the extracellular domain, which trigger different signaling cascades [48-51]. Considering the PEA3-rearrangement context and the androgen-regulated 
nature of the TMEFF2 gene, it would be interesting to evaluate how the activity of androgens can modulate the expression, subcellular localization and signaling of TMEFF2 isoforms under PEA3 overexpression. This discrepancy between the observed in vitro and in vivo expression profiles is also patent in the absence of shared candidate targets between in vitro ETV1-associated genes here identified and the in vivo ETV1-associated genes previously identified [23]. However, as shown by the interaction network analysis, several of the in vitro ETV1 candidate target genes may act in similar pathways as the tumor-associated ETV1 candidate target genes by shared intermediate partners, once again suggesting the importance of the environmental factors and of the cellular context in the activity of key molecular players, such as the ETS transcription factors.

Despite the consistent reports on the involvement of either ETV1 or ETV4 in invasion and AIG in vitro, and comparing to what is accepted for other tumor types $[42,52]$, validation of the contribution of these PEA3 members to an increased aggressiveness of prostate tumors is only emerging. In fact, very few reports clearly suggested that association. In 2008, Attard et al. described ETV1 rearrangements in 23 cases, which were shown to be associated with higher pathological staging, Gleason score and PSA [9]. In 2012, Shaikhibrahim et al. reported that ERG and ETVI are up-regulated in the glands of the peripheral zone comparing with the transitional zone of the prostate, and that PEA3 rearrangements can occur de novo in metastatic lesions of rearrangement-negative primary tumors (contrarily to ERG rearrangements) [30, 53]. Searching for additional validation, we used data from GSE26242 to look for differentially expressed genes using the interactive web tool GEO2R from the National Center for Bioinformatics (NCBI) [54]. In this cohort, ETV1 and ETV4 expression, but not $E R G$, were associated $(p<0.05)$ with higher pathological staging ( $\geq \mathrm{pT} 3$ versus $\leq \mathrm{pT} 2$ ), with ETV1 also associated with higher Gleason score (GS $\geq 8$ versus $\mathrm{GS} \leq 6$ ) (Supplementary Figure 1$)$. Nevertheless, even though one can prove that a specific gene is regulated by a certain transcription factor, the specificity of that regulation is less plausible, as the same gene can be regulated by other proteins or transcription factors, depending on the cellular context. So, we questioned whether we could use the in vitro cellular models with impact in AIG and invasion to find molecular players that may play a role in tumor aggressiveness in vivo, independently of the ETS background. By crossing the expression profiles of the cellular models that showed the same phenotypic impact and excluding those that did not, we defined lists of candidate molecular players in AIG and invasion. Interestingly, despite the absence of an overlap between the ETV1associated in vitro and in vivo gene sets, two of the genes associated with invasion and/or AIG, namely, CDK19 and PROS1, are present in our in vivo gene set, increasing their potential to be involved in the aggressiveness of tumors with ETV1-rearrangement (Supplementary Tables 3 and 4). Although overall the links between the "invasion and/ or AIG" genes and prostate cancer or carcinogenesis in general are scarce, the association of decreased SLC22A3 levels and tumor aggressiveness was previously reported by two groups as part of both an "underexpressed in high Gleason grade signature" and an "underexpressed in progression signature" $[55,56]$. Interestingly, SLC22A3 is also included in our list of ETV4-specific candidate target genes, showing inverse correlation with ETV4 expression. Since there are no large series of ETV4-positive tumors that can be useful in the evaluation of the prognostic value of ETV4-rearrangements or of the correlation between these and the expression of $S L C 22 A 3$, further studies are necessary to evaluate SLC22A3 link with ETV4 and its involvement in AIG. In the study from Tomlins et al., decreased GSTM4 expression was also associated with disease progression as part of the "glutathione metabolism pathway" [55]. Although none of the genes that we found associated with Gleason score and pathological staging in our series was present in the panel of 255 genes evaluated in GSE26242, in the same study Long et al. further showed a panel of 304 genes that differentiate tumors with $\mathrm{GS}=7(3+4)$ from tumors with $\mathrm{GS}=7(4+3)$, where the gene $A T F 3$, also identified in our analysis, was described to be significantly decreased in the group of tumors with higher Gleason score [57]. Several other research groups have proposed gene panels that discriminate aggressive from non-aggressive prostate carcinomas [58] and differences between them may be related to several aspects, ranging from characteristics of the sample cohorts to differences in methods and analysis of the data. To our knowledge this is the first study that aimed to translate information from in vitro to in vivo datasets in the context of PEA3 overexpression. The potential of the panel of seven genes here described as associated with pT3 tumors and their involvement in invasion and/or AIG in the context of PEA3 overexpression warrants further investigation.

In summary, this study provides new information on the oncogenic role of ETV1 and ETV4 in prostate carcinogenesis by looking at a co-expression cellular context in vitro. We show that these ETS transcription factors have partially overlapping functions, with ETV1 being more relevant for cell invasion and ETV4 for anchorage-independent growth. At the molecular level, expression signatures of each ETS reveal the regulation of both specific and shared candidate targets that may resemble cellular mechanisms in vivo by interaction with the same intermediate partners. We further identified a panel of genes associated with invasion and anchorageindependent growth mediated by ETV1 and ETV4 in vitro that may be useful as prognostic markers, independently of the ETS rearrangement status. Larger cohorts would be valuable in the validation of these markers and of their link with PEA3 rearrangements, eventually allowing outlining a subset of tumors with worse prognosis. 


\section{METHODS}

\section{Cell culture}

PC3 and LNCaP cells were acquired from the German Resource Centre for Biological Material (DSMZ, Braunschweig, Germany). VCaP and PNT2 cells were acquired from The European Collection of Cell Cultures (Sigma-Aldrich, St. Louis, MO). 22Rv1 cells were kindly provided by Dr. David Sidransky at the Johns Hopkins University School of Medicine. MDAPCa-2b and NCI-H660 cells were kindly provided by Prof. Ragnhild A. Lothe from the Department of Cancer Prevention at the Institute for Cancer Research, Oslo University Hospital, Norway, and TPC-1 cell line was kindly provided by Prof. Paula Soares from the Cancer Biology Group at the Institute of Molecular Pathology and Immunology of the University of Porto. The virus packaging RetroPack ${ }^{\mathrm{TM}}$ PT67 cell line was acquired from Clontech Laboratories, Inc. (Saint-Germain-enLaye, France). Human PC3 cells were grown in F-12 medium, LNCaP, PNT2, 22Rv1, TPC-1 and NCI-H660 cells were grown in RPMI 1640, and VCaP and PT67 cells were grown in DMEM, all from GIBCO, by Life Technologies (Carlsbad, CA). MDA-PCa-2b cells were cultured in BRFF-HPC1 medium (Gentaur, Brussels, Belgium). All media were supplemented with $10 \%$ fetal bovine serum (FBS) and 1\% penicillin/streptomycin, both from GIBCO, with exception of MDA-PCa-2b cells which were supplemented with $20 \%$ FBS, and NCI-H660 cells, supplemented with 5\% FBS, $1 \mathrm{X}$ Insulin-transferrinselenium (GIBCO), $2 \mathrm{mM}$ L-glutamine (GIBCO), $10 \mathrm{nM}$ Hydrocortisone (Sigma-Aldrich), and $10 \mathrm{nM}$ betaestradiol (Sigma-Aldrich). Cells were maintained under appropriate growth conditions. Conventional G-banding karyotyping was performed to confirm cell identity and all prostate cell lines were routinely tested for Mycoplasma spp. contamination (PCR Mycoplasma Detection Set, Clontech Laboratories Inc.).

\section{Taqman low density array (TLDA)}

To evaluate the expression of different ETS transcription factors (ERG, ETV1, ETV4, ETV5 and FLI1), all prostate cell lines were analyzed using a TLDA card from Applied Biosystems (by Life Technologies, Foster City, CA) [6]. Briefly, after RNA extraction with TRIzol ${ }^{\circledR}$ Reagent (Invitrogen by Life Technologies), 100ug of RNA were converted to cDNA using the High-Capacity RNA-to-cDNA kit (Applied Biosystems), according to the manufacturer's instructions. The triplicate well format TLDA card included manufacturers' pre-developed probes for ERG (Hs01554635_m1), ETV1 (Hs00951941_m1), ETV4 (Hs00385910_m1), ETV5 (Hs00231790_m1), FLII (Hs00956711_m1), GUSB (Hs99999908_m1) and 18S (as pre-included housekeeping control). ETS expression values were obtained by the Comparative Ct method [59], using average $18 S$ and $G U S B$ expression as normalization controls. To validate true ETS outlier expression, ETS expression from each cell line was normalized to the median ETS expression found in our series of prostate carcinomas [6]. Average expression values of $91 \mathrm{PCa}$ with $E R G$ rearrangement, 12 with ETV1 rearrangement, three with ETV4 rearrangement, one with ETV5 rearrangement, one with FLII rearrangement and 78 without known ETS rearrangements were used for comparison.

\section{Fluorescence in situ hybridization (FISH)}

To look for genomic rearrangements involving the ETV1 and ETV4 transcription factors, we performed FISH in PC3, MDA-PCa-2b and LNCaP cells. Bacterial artificial chromosomes (BACs) were selected using the (UCSC) Human Genome Browser and were obtained from the BACPAC Resource Center (Oakland, CA). For the detection of the ETVI gene, the BAC clones used were RP11-941F13 and RP11-790J2 (at 5' and 3', respectively), whereas the BACs RP11-259G18 and RP11-831F13 were used to target the ETV4 gene (at 5' and 3', respectively). BAC DNA was isolated, amplified and labeled as previously described [60]. The integrity and correct localization of all probes was verified on normal human metaphases.

\section{Generation of cell line models}

Stable silencing of ETV1 in LNCaP cells was previously described [23]. The same approach was used for stable silencing of ETVI and ETV4 in MDA-PCa-2b and PC 3 cells. Briefly, two shRNA sequences against ETV1 or ETV4 were selected and designed using the RNAi Target Sequence Selector and the shRNA Sequence Designer, respectively (Supplementary Table 5). Both shETV1 and shETV4 sequences were cloned into the pSIREN-Retro-Q vector (Clontech Laboratories Inc.). A negative control vector carrying a nontargeting sequence (pSIREN-shNeg) was also generated (Protocol No. PT3132-1, Version No. PR631543). After transfection into the PT67 packaging cell line, viral medium was harvested and used to infect the cells of interest. Stably silenced cell populations were obtained by puromycin selective pressure $(1 \mu \mathrm{g} / \mathrm{mL}$, Clontech Laboratories Inc.). The cell populations showing lower ETV1 or ETV4 expression levels (evaluated by qRT-PCR, not shown) were selected (shETV1-553 cells from MDA-PCa-2b, shETV1-1037 cells from LNCaP and PC3, and shETV4-664 cells from both MDA-PCa-2b and PC3) and clonal populations were isolated using the serial dilutions technique. At least six clonal populations were isolated for each condition and two independent clones showing low levels of the silenced ETS (evaluated by qRT-PCR, not shown) were selected for further studies. For each cell line, a control population carrying a non-targeting sequence (shNeg) and two independent low ETV1 or ETV4 expressing clones (shETV1-C1 and shETV1-C2, 
or shETV4-C1 and shETV4-C2, respectively) were used. For the MDA-PCa-2b shETV1 cells, we were not able to establish clonal populations, thus two independent polyclonal populations were selected (shETV1-P1 and shETV1-P2). De novo overexpression of ETV1 in PNT2 cells was previously described [23]. Briefly, the fulllength coding sequence of ETV1 (ENST00000242066) was amplified from LNCaP cells and cloned into the pMSCVneo vector (Clontech Laboratories Inc.) using the In-Fusion Cloning System (Clontech Laboratories Inc.). PT67 cells were transfected with pMSCV constructs and with the empty vector pMSCVneo and harvested viral media were used to infect PNT2 cells. Transfected PNT2 cells were expanded under G418 selective pressure (300 $\mu \mathrm{g} / \mathrm{mL}, \mathrm{GIBCO})$. A control population (PNT2-Neo) and one cell population showing overexpression of fulllength ETV1 (PNT2-ETV1) were used.

\section{Quantitative reverse transcription PCR (qRT-PCR)}

To evaluate the expression levels of ETV1 and ETV4 in the established cell line models with silencing of these PEA3 members, total RNA was extracted from all cell populations using the Illustra TriplePrep kit (GE Healthcare, Cleveland, USA) and cDNA was synthesized using the H-minus RevertAid cDNA synthesis kit (Fermentas, St. Leon-Rot, Germany) with oligo-dT primers, according to the manufacturers' protocol. Primers and probes were either acquired as pre-developed TaqMan Gene Expression Assays from Applied Biosystems or designed using the software Primer Express and acquired from Metabion (Martinsried, Germany) (Supplementary Table 6). The beta-glucuronidase (GUSB) housekeeping gene was used as an endogenous control for normalization of the expression levels. Relative expression levels of each ETS were obtained by calibrating GUSB normalized ETS expression values from each population for the expression levels of the corresponding control population (shNeg).

\section{Western-blotting}

Protein fractions were obtained from subconfluent cells after RNA extraction with the Illustra TriplePrep kit (mentioned above). Concentration was measured by the Pierce BCA protein assay (Thermo Fisher Scientific, Rockford, USA), according to the manufacturer's instructions. For the immunoblotting, $30 \mu \mathrm{g}$ of protein extracts were run on a $10 \%$ SDS-PAGE gel and blotted onto a Protran nitrocellulose membrane (GE Healthcare). Protein detection was accomplished by overnight incubation at $4^{\circ} \mathrm{C}$, using the following primary antibodies: mouse anti-ETV4 (1:500, clone 3G9-1B9, H00002118-M01, Abnova, Atlanta, USA); mouse antiETV1 (1:500, clone 4C12, SAB1403794, Sigma-Aldrich) and mouse anti- $\beta$-actin (1:8000, clone AC-15, A1978, Sigma-Aldrich) monoclonal antibodies, respectively. An anti-mouse horseradish peroxidase-conjugated secondary antibody was used (1:2500, Santa Cruz Biotechnology, Heidelberg, Germany) and signals were detected using the Immun-Star WesternC Chemiluminescent kit (Bio-Rad Laboratories, Inc., Munich, Germany).

\section{Proliferation assay}

Assessment of cell viability was performed using the MTT assay (Sigma-Aldrich). Cells were seeded onto 96well plates (Sarstedt, Nümbrecht, Germany) at specific cell densities (Supplementary Table 7) and incubated at normal growth conditions for 24, 48, 72 and $96 \mathrm{hrs}$. Twelve to 24 hrs after cell seeding (depending on the cell line), a time zero measure was performed, corresponding to the cell viability measure of post-adherence, pre-proliferating, seeded cells. At each time point (including time zero), $100 \mu \mathrm{L}$ of $0.5 \mathrm{mg} / \mathrm{mL}$ MTT solution were added and cells were incubated for $2 \mathrm{hrs}$ (PC3 and LNCaP) or 30 min (MDA-PCa-2b). Formazan crystals were solubilized with $50 \mu \mathrm{L}$ of dimethyl sulfoxide (DMSO, Merck). The optical density was measured using a microplate reader (Fluostar Omega, BMG Labtech, Ortenberg, Germany) at a wavelength of $540 \mathrm{~nm}$ with background correction at $630 \mathrm{~nm}$. For each time-point, an average value of measures from nine replicate wells was obtained. Cell population growth was estimated by correcting and normalizing the average absorbance values obtained in each time-point (Tn) to the average absorbance values of the time zero (T0) by the following formula: (Tn-T0)/ T0. Relative growth was obtained by normalizing values of each silenced cell population to its respective control. Three independent assays were performed.

\section{Apoptosis assay}

Apoptosis was analyzed using the APOPercentage apoptosis assay kit (Biocolor, Carrickfergus, UK) according to the manufacturer's instructions. Cells were seeded onto 96-well plates (Sarstedt) at specific cell densities (Supplementary Table 7) and incubated at normal growth conditions for $96 \mathrm{hrs}$. The absorbance was determined using a microplate reader (Fluostar Omega) at $550 \mathrm{~nm}$ with background correction at $620 \mathrm{~nm}$. An average value of measures from nine replicate wells was obtained for each cell population. Relative apoptosis was obtained by normalizing values of each silenced cell population to its respective control. Three independent assays were performed.

\section{Anchorage-independent growth (AIG)}

AIG was measured using the soft agar colony formation assay. A $0.6 \%$ bottom layer of low melting point agarose (Lonza by VWR, Radnor, EUA) in normal growth medium was prepared in six-well culture plates 
(Sarstedt). On top, a layer of $0.2 \%$ agarose containing cells (Supplementary Table 7) was placed and covered with culture medium. Cells were incubated at normal growth conditions for two to three weeks. Photographs of representative fields were taken and colonies containing more than seven cells were considered for counting. Relative aggregation was obtained by normalizing values of each silenced cell population to its respective control. Three independent assays were performed.

\section{Invasion assay}

Cell invasion was evaluated using the Oris ${ }^{\mathrm{TM}}$ Cell Invasion \& Detection Assay kit (Platypus Technologies, Madison, USA) and the BD BioCoat ${ }^{\mathrm{TM}}$ Matrigel $^{\mathrm{TM}}$ Invasion Chamber (BD Biosciences), according to the manufacturer's recommendations. The first was used with PC3 and PNT2 derived cells, whereas the second was used with LNCaP derived cells. Both assays were used with MDA-PCa-2b derived cells and with the TPC-1 cell line, as a positive control. For the Oris ${ }^{\mathrm{TM}}$ Cell Invasion assay, cells were seeded at specific cell densities (Supplementary Table 7) and incubated at normal growth conditions for eight days. At this time-point, cells were stained with Calcein AM (AMS Biotechnology, Abingdon, UK) at a final concentration of $0.5 \mu \mathrm{g} / \mathrm{mL}$. A detection mask was attached to the plate bottom to restrict visualization of the detection area and measurements were made in a microplate reader (Fluostar Omega). For the Boyden chambers, cell suspensions in serum-free medium (Supplementary Table 7) were loaded in the upper chambers and complete medium was added to the lower chamber. After $48 \mathrm{hrs}$ incubation at normal growth conditions, the non-invading cells attached to the upper surface were removed with cotton swabs, and invading cells on the lower side of the membrane were stained with DAPI and visualized in a fluorescence microscope (Olympus Corporation, Tokyo, Japan). Photographs of representative fields were taken using a $10 \mathrm{x}$ objective lens and the cellSens Dimension software (Olympus Corporation) was used for counting. Relative cell invasion was obtained by normalizing values of each silenced cell population to its respective control. Three independent assays were performed.

\section{Gene expression profile}

The GeneChip Human Exon 1.0 ST arrays were used to assess whole-transcriptome expression levels of the different cell line models. The expression profile of each cell population was obtained after background correction and normalization in the Affymetrix Expression Console v1.1 software, using the Robust Multichip Average (RMA) algorithm. Considering the lower expression of ETV1 in PC3 cells comparing with those of MDA-PCa-2b and LNCaP cells, the shETV1-PC3 cells were not evaluated. Two independent populations of the PNT2-ETV1 cells were evaluated together with the PNT2-Neo control. For each gene and each cell line, two fold-change values, from two manipulated cell populations versus control, were obtained. Whenever a gene showed the same differential expression profile (increased or decreased with ETS expression) in both manipulated cell populations, the average fold-change was calculated and the gene was considered for subsequent filtering.

\section{Identification of specific and shared ETV1 and ETV4 target genes in vitro}

To identify ETV1 and/or ETV4 candidate target genes, the expression profiles of the cell populations sharing the overexpressed ETS were used. Considering the non-tumorigenic nature of the PNT2 cell line, the expression profile of the PNT2-ETV1 cells was not considered for target filtering. To look for ETS-specific candidate target genes we selected those that showed to be up or down-regulated no less than 1.5-fold in the two cell line models where the expression of the same ETS was silenced (LNCaP and MDA-PCa-2b cells for ETV1, and PC3 and MDA-PCa-2b cells for ETV4). As for the target genes shared by ETV1 and ETV4, we selected those that showed to be up or down-regulated at least 1.5-fold in three of the four silenced cell lines (LNCaP-shETV1, MDA-PCa2b-shETV1, PC3-shETV4 and MDA-PCa-2b-shETV4).

\section{Gene set enrichment and interaction network analyses}

To explore the functional classification of the candidate target genes obtained by differential expression analysis (as explained above) we used the ConsensusPathDB, which integrates protein interaction information from 32 public interaction databases (Release 29, 27.06.2014) [61-63]. To evidence specific and shared features of ETV1 and ETV4 candidate target genes, analyses of enrichment of "gene ontology categories" and "gene involvement in known signalling pathways" were performed with the full list of PEA3 candidate targets $(n=61)$. A cut-off $p$ value of 0.02 was used in both analyses. To search for pathway-based sets the information from all ConsensusPathDB integrated databases was considered. To explore possible proteinprotein interaction networks associated with the gene sets identified in vitro, the "induced network module" tool of the ConsensusPathDB database was used. High and medium-confidence, binary and complex, proteinprotein interactions and biochemical reactions were selected for filtering. Considering our previous work focusing on the identification of candidate target genes of ERG and ETV1 using a panel of PCa subtyped for ETS rearrangements [23], we questioned whether in vitro and in vivo ETV1 candidate targets would overlap or be involved in the same signaling pathways. We thus performed the interaction network analysis using the full 
list of ETV1 candidate target genes in vitro and in vivo (Supplementary Table 3).

\section{Identification of invasion and/or AIG associated genes in vitro}

To find gene sets associated with the observed impact of ETV1 and ETV4 overexpression in invasion and AIG that may be useful as markers of tumor aggressiveness in vivo we crossed the gene expression profiles of the cell line models showing impact in these phenotypic characteristics. To identify genes involved in invasion and/or AIG, we looked for genes that showed the same differential expression profile in the three cell line models showing equal impact in both phenotypic characteristics - shETV4-PC3, shETV1-LNCaP and PNT2-ETV1 cell lines (fold-change higher than 1.2) - and excluded those differentially expressed in the cell line model showing no impact in both phenotypes (shETV1-MDA-PCa-2b cells, fold-change higher than 1.2). Since we observed more involvement of ETV4 in AIG, we looked for genes with higher potential to be involved in this phenotypic characteristic by selecting those that were differentially expressed (fold-change higher than 1.5) in the shETV4 populations of both the MDA-PCa-2b and PC3 cells and excluded those that showed the same differential expression (foldchange higher than 1.2) in the shETV1 populations of the MDA-PCa-2b cells. Similarly, since we observed more involvement of ETV1 in invasion, we looked for genes with higher potential to be involved in invasion by selecting those that were differentially expressed (foldchange higher than 1.5) in both shETV1-LNCaP and PNT2-ETV1 cell populations, and excluded those that showed the same expression profile (fold-change higher than 1.2) in both the shETV4 and shETV1 populations of the MDA-PCa-2b cells.

\section{In vivo validation and clinico-pathological associations}

To evaluate whether the observed in vitro associations between ETV1 and ETV4 overexpression and the candidate target genes would be useful in the identification of $\mathrm{PCa}$ harboring rearrangements of these ETS members, and to evaluate the usefulness of the sets of genes associated with invasion and AIG in the identification of aggressive prostate carcinomas, we used our exon-array expression data from a series of nine noncancerous prostate tissues (NPT) and 50 prostate carcinomas $(\mathrm{PCa})$ subtyped for ETS rearrangements [23], which include $14 \mathrm{PCa}$ with PEA3 rearrangements (12 of which with ETV1 rearrangement), $22 \mathrm{PCa}$ with ERG rearrangement and $14 \mathrm{PCa}$ without known ETS rearrangements. To search for clinico-pathological associations, information on age at diagnosis, PSA at diagnosis, pathological staging and Gleason score was used [6].

\section{Statistical analysis}

All results from in vitro assays are expressed as a ratio of the data obtained with each clone and the scramble control, from three independent experiments, each including triplicate wells per condition. The Students $t$-test was used to assess the significance of the differences. To compare expression levels between ETS groups and to find clinico-pathological associations, the Mann-Whitney test was used. To evaluate the performance of the panel of seven genes as biomarkers for the identification of tumors with local invasion using different cut-off values, the Pearson Chi-square test was used. In every statistical test, a $p$ value smaller than 0.05 was considered statistically significant.

\section{ACKNOWLEDGMENTS}

This work was supported by the Portuguese Oncology Institute of Porto (CI-IPOP-9-2008 and CI-IPOP-16-2012) and partly supported by the Research Council of Norway through its Centers of Excellence funding scheme (project number 179571). DM and PP are research fellows from Liga Portuguesa Contra o Cancro, Núcleo Regional do Norte (MT). JDBS (SFRH/ $\mathrm{BD} / 46574 / 2008)$ and JS (SFRH/BD/73964/2010) are research fellows from FCT.

\section{Abbreviations}

AIG, anchorage-independent growth; DMSO, dimethyl sulfoxide; FBS, fetal bovine serum; FISH, fluorescence in situ hybridization; MTT, 3-(4,5-dimethylthiazol-2-yl)-2,5diphenyltetrazolium bromide; NPT, noncancerous prostate tissues; $\mathrm{PCa}$, prostate carcinoma; qRT-PCR, quantitative reverse transcription polymerase chain reaction; shRNA, short hairpin RNA; TLDA, TaqMan low density array.

\section{REFERENCES}

1. Rubin MA, Maher CA, Chinnaiyan AM. Common gene rearrangements in prostate cancer. Journal of clinical oncology : official journal of the American Society of Clinical Oncology. 2011; 29:3659-3668.

2. Kumar-Sinha C, Tomlins SA, Chinnaiyan AM. Recurrent gene fusions in prostate cancer. Nature reviews Cancer. 2008; 8:497-511.

3. Helgeson BE, Tomlins SA, Shah N, Laxman B, Cao Q, Prensner JR, Cao X, Singla N, Montie JE, Varambally S, Mehra R, Chinnaiyan AM. Characterization of TMPRSS2:ETV5 and SLC45A3:ETV5 gene fusions in prostate cancer. Cancer research. 2008; 68:73-80. 
4. Tomlins SA, Mehra R, Rhodes DR, Smith LR, Roulston D, Helgeson BE, Cao X, Wei JT, Rubin MA, Shah RB, Chinnaiyan AM. TMPRSS2:ETV4 gene fusions define a third molecular subtype of prostate cancer. Cancer research. 2006; 66:3396-3400.

5. Tomlins SA, Rhodes DR, Perner S, Dhanasekaran SM, Mehra R, Sun XW, Varambally S, Cao X, Tchinda J, Kuefer R, Lee C, Montie JE, Shah RB, et al. Recurrent fusion of TMPRSS2 and ETS transcription factor genes in prostate cancer. Science. 2005; 310:644-648.

6. Paulo P, Barros-Silva JD, Ribeiro FR, Ramalho-Carvalho J, Jeronimo C, Henrique R, Lind GE, Skotheim RI, Lothe RA, Teixeira MR. FLI1 is a novel ETS transcription factor involved in gene fusions in prostate cancer. Genes, chromosomes \& cancer. 2012; 51:240-249.

7. Barros-Silva JD, Paulo P, Bakken AC, Cerveira N, Lovf M, Henrique R, Jeronimo C, Lothe RA, Skotheim RI, Teixeira MR. Novel 5' fusion partners of ETV1 and ETV4 in prostate cancer. Neoplasia. 2013; 15:720-726.

8. Hermans KG, Bressers AA, van der Korput HA, Dits NF, Jenster G, Trapman J. Two unique novel prostate-specific and androgen-regulated fusion partners of ETV4 in prostate cancer. Cancer research. 2008; 68:3094-3098.

9. Attard G, Clark J, Ambroisine L, Mills IG, Fisher G, Flohr P, Reid A, Edwards S, Kovacs G, Berney D, Foster C, Massie CE, Fletcher A, et al. Heterogeneity and clinical significance of ETV1 translocations in human prostate cancer. British journal of cancer. 2008; 99:314-320.

10. Han B, Mehra R, Dhanasekaran SM, Yu J, Menon A, Lonigro RJ, Wang X, Gong Y, Wang L, Shankar S, Laxman B, Shah RB, Varambally S, et al. A fluorescence in situ hybridization screen for E26 transformation-specific aberrations: identification of DDX5-ETV4 fusion protein in prostate cancer. Cancer research. 2008; 68:7629-7637.

11. Cerveira N, Ribeiro FR, Peixoto A, Costa V, Henrique R, Jeronimo C, Teixeira MR. TMPRSS2-ERG gene fusion causing ERG overexpression precedes chromosome copy number changes in prostate carcinomas and paired HGPIN lesions. Neoplasia. 2006; 8:826-832.

12. Tomlins SA, Laxman B, Varambally S, Cao X, Yu J, Helgeson BE, Cao Q, Prensner JR, Rubin MA, Shah RB, Mehra R, Chinnaiyan AM. Role of the TMPRSS2ERG gene fusion in prostate cancer. Neoplasia. 2008; 10:177-188.

13. Klezovitch O, Risk M, Coleman I, Lucas JM, Null M, True LD, Nelson PS, Vasioukhin V. A causal role for ERG in neoplastic transformation of prostate epithelium. Proceedings of the National Academy of Sciences of the United States of America. 2008; 105:2105-2110.

14. King JC, Xu J, Wongvipat J, Hieronymus H, Carver BS, Leung DH, Taylor BS, Sander C, Cardiff RD, Couto SS, Gerald WL, Sawyers CL. Cooperativity of TMPRSS2-ERG with PI3-kinase pathway activation in prostate oncogenesis. Nature genetics. 2009; 41:524-526.
15. Carver BS, Tran J, Gopalan A, Chen Z, Shaikh S, Carracedo A, Alimonti A, Nardella C, Varmeh S, Scardino PT, Cordon-Cardo C, Gerald W, Pandolfi PP. Aberrant ERG expression cooperates with loss of PTEN to promote cancer progression in the prostate. Nature genetics. 2009; 41:619-624.

16. Sharrocks AD. The ETS-domain transcription factor family. Nature reviews Molecular cell biology. 2001; 2:827-837.

17. Svensson MA, LaFargue CJ, MacDonald TY, Pflueger D, Kitabayashi N, Santa-Cruz AM, Garsha KE, Sathyanarayana UG, Riley JP, Yun CS, Nagy D, Kosmeder JW, Pestano GA, et al. Testing mutual exclusivity of ETS rearranged prostate cancer. Laboratory investigation; a journal of technical methods and pathology. 2011; 91:404-412.

18. Wang J, Cai Y, Yu W, Ren C, Spencer DM, Ittmann M. Pleiotropic biological activities of alternatively spliced TMPRSS2/ERG fusion gene transcripts. Cancer research. 2008; 68:8516-8524.

19. Sun C, Dobi A, Mohamed A, Li H, Thangapazham RL, Furusato B, Shaheduzzaman S, Tan SH, Vaidyanathan G, Whitman E, Hawksworth DJ, Chen Y, Nau M, et al. TMPRSS2-ERG fusion, a common genomic alteration in prostate cancer activates C-MYC and abrogates prostate epithelial differentiation. Oncogene. 2008; 27:5348-5353.

20. Firlej V, Ladam F, Brysbaert G, Dumont P, Fuks F, de Launoit Y, Benecke A, Chotteau-Lelievre A. Reduced tumorigenesis in mouse mammary cancer cells following inhibition of Pea3- or Erm-dependent transcription. Journal of cell science. 2008; 121:3393-3402.

21. Hollenhorst PC, Jones DA, Graves BJ. Expression profiles frame the promoter specificity dilemma of the ETS family of transcription factors. Nucleic acids research. 2004; 32:5693-5702.

22. Maroulakou IG, Bowe DB. Expression and function of Ets transcription factors in mammalian development: a regulatory network. Oncogene. 2000; 19:6432-6442.

23. Paulo P, Ribeiro FR, Santos J, Mesquita D, Almeida M, Barros-Silva JD, Itkonen $\mathrm{H}$, Henrique R, Jeronimo C, Sveen A, Mills IG, Skotheim RI, Lothe RA, et al. Molecular subtyping of primary prostate cancer reveals specific and shared target genes of different ETS rearrangements. Neoplasia. 2012; 14:600-611.

24. Cai C, Hsieh CL, Omwancha J, Zheng Z, Chen SY, Baert JL, Shemshedini L. ETV1 is a novel androgen receptor-regulated gene that mediates prostate cancer cell invasion. Molecular endocrinology. 2007; 21:1835-1846.

25. Hermans KG, van der Korput HA, van Marion R, van de Wijngaart DJ, Ziel-van der Made A, Dits NF, Boormans JL, van der Kwast TH, van Dekken H, Bangma CH, Korsten H, Kraaij R, Jenster G, et al. Truncated ETV1, fused to novel tissue-specific genes, and full-length ETV1 in prostate cancer. Cancer research. 2008; 68:7541-7549. 
26. Tomlins SA, Laxman B, Dhanasekaran SM, Helgeson BE, Cao X, Morris DS, Menon A, Jing X, Cao Q, Han B, Yu J, Wang L, Montie JE, et al. Distinct classes of chromosomal rearrangements create oncogenic ETS gene fusions in prostate cancer. Nature. 2007; 448:595-599.

27. Hollenhorst PC, Paul L, Ferris MW, Graves BJ. The ETS gene ETV4 is required for anchorage-independent growth and a cell proliferation gene expression program in PC3 prostate cells. Genes \& cancer. 2011; 1:1044-1052.

28. Pellecchia A, Pescucci C, De Lorenzo E, Luceri C, Passaro N, Sica M, Notaro R, De Angioletti M. Overexpression of ETV4 is oncogenic in prostate cells through promotion of both cell proliferation and epithelial to mesenchymal transition. Oncogenesis. 2012; 1:e20.

29. Braun M, Goltz D, Shaikhibrahim Z, Vogel W, Bohm D, Scheble V, Sotlar K, Fend F, Tan SH, Dobi A, Kristiansen G, Wernert N, Perner S. ERG protein expression and genomic rearrangement status in primary and metastatic prostate cancer--a comparative study of two monoclonal antibodies. Prostate Cancer Prostatic Dis. 2012; 15:165-169.

30. Shaikhibrahim Z, Braun M, Nikolov P, Boehm D, Scheble V, Menon R, Fend F, Kristiansen G, Perner S, Wernert N. Rearrangement of the ETS genes ETV1, ETV-4, ETV-5, and ELK-4 is a clonal event during prostate cancer progression. Human pathology. 2012; 43:1910-1916.

31. Aytes A, Mitrofanova A, Kinkade CW, Lefebvre C, Lei M, Phelan V, LeKaye HC, Koutcher JA, Cardiff RD, Califano A, Shen MM, Abate-Shen C. ETV4 promotes metastasis in response to activation of PI3-kinase and Ras signaling in a mouse model of advanced prostate cancer. Proceedings of the National Academy of Sciences of the United States of America. 2013; 110:E3506-3515.

32. Baena E, Shao Z, Linn DE, Glass K, Hamblen MJ, Fujiwara Y, Kim J, Nguyen M, Zhang X, Godinho FJ, Bronson RT, Mucci LA, Loda M, et al. ETV1 directs androgen metabolism and confers aggressive prostate cancer in targeted mice and patients. Genes \& development. 2013; 27:683-698.

33. Morris DS, Tomlins SA, Montie JE, Chinnaiyan AM. The discovery and application of gene fusions in prostate cancer. BJU international. 2008; 102:276-282.

34. Tomlins SA, Bjartell A, Chinnaiyan AM, Jenster G, Nam RK, Rubin MA, Schalken JA. ETS gene fusions in prostate cancer: from discovery to daily clinical practice. European urology. 2009; 56:275-286.

35. Tai S, Sun Y, Squires JM, Zhang H, Oh WK, Liang CZ, Huang J. PC3 is a cell line characteristic of prostatic small cell carcinoma. Prostate. 2011; 71:1668-1679.

36. Gasi D, van der Korput HA, Douben HC, de Klein A, de Ridder CM, van Weerden WM, Trapman J. Overexpression of full-length ETV1 transcripts in clinical prostate cancer due to gene translocation. PloS one. 2011; 6:e16332.
37. Clementz AG, Rogowski A, Pandya K, Miele L, Osipo C. NOTCH-1 and NOTCH-4 are novel gene targets of PEA3 in breast cancer: novel therapeutic implications. Breast Cancer Res. 2011; 13:R63.

38. Jane-Valbuena J, Widlund HR, Perner S, Johnson LA, Dibner AC, Lin WM, Baker AC, Nazarian RM, Vijayendran KG, Sellers WR, Hahn WC, Duncan LM, Rubin MA, et al. An oncogenic role for ETV1 in melanoma. Cancer research. 2010; 70:2075-2084.

39. $\mathrm{Xu} \mathrm{LL}$, Stackhouse BG, Florence $\mathrm{K}$, Zhang $\mathrm{W}$, Shanmugam N, Sesterhenn IA, Zou Z, Srikantan V, Augustus M, Roschke V, Carter K, McLeod DG, Moul JW, et al. PSGR, a novel prostate-specific gene with homology to a $\mathrm{G}$ protein-coupled receptor, is overexpressed in prostate cancer. Cancer research. 2000; 60:6568-6572.

40. Neuhaus EM, Zhang W, Gelis L, Deng Y, Noldus J, Hatt H. Activation of an olfactory receptor inhibits proliferation of prostate cancer cells. The Journal of biological chemistry. 2009; 284:16218-16225.

41. Kristiansen K. Molecular mechanisms of ligand binding, signaling, and regulation within the superfamily of G-protein-coupled receptors: molecular modeling and mutagenesis approaches to receptor structure and function. Pharmacology \& therapeutics. 2004; 103:21-80.

42. Oh S, Shin S, Janknecht R. ETV1, 4 and 5: an oncogenic subfamily of ETS transcription factors. Biochimica et biophysica acta. 2012; 1826:1-12.

43. Ohnishi J, Ohnishi E, Jin M, Hirano W, Nakane D, Matsui H, Kimura A, Sawa H, Nakayama K, Shibuya H, Nagashima $\mathrm{K}$, Takahashi T. Cloning and characterization of a rat ortholog of MMP-23 (matrix metalloproteinase-23), a unique type of membrane-anchored matrix metalloproteinase and conditioned switching of its expression during the ovarian follicular development. Molecular endocrinology. 2001; 15:747-764.

44. Gery S, Sawyers CL, Agus DB, Said JW, Koeffler HP. TMEFF2 is an androgen-regulated gene exhibiting antiproliferative effects in prostate cancer cells. Oncogene. 2002; 21:4739-4746.

45. Liang G, Robertson KD, Talmadge C, Sumegi J, Jones PA. The gene for a novel transmembrane protein containing epidermal growth factor and follistatin domains is frequently hypermethylated in human tumor cells. Cancer research. 2000; 60:4907-4912.

46. Afar DE, Bhaskar V, Ibsen E, Breinberg D, Henshall SM, Kench JG, Drobnjak M, Powers R, Wong M, Evangelista F, O'Hara C, Powers D, DuBridge RB, et al. Preclinical validation of anti-TMEFF2-auristatin E-conjugated antibodies in the treatment of prostate cancer. Molecular cancer therapeutics. 2004; 3:921-932.

47. Glynne-Jones E, Harper ME, Seery LT, James R, Anglin I, Morgan HE, Taylor KM, Gee JM, Nicholson RI. TENB2, a proteoglycan identified in prostate cancer that is associated with disease progression and androgen independence. International journal of cancer Journal international du cancer. 2001; 94:178-184. 
48. Ali N, Knauper V. Phorbol ester-induced shedding of the prostate cancer marker transmembrane protein with epidermal growth factor and two follistatin motifs 2 is mediated by the disintegrin and metalloproteinase-17. The Journal of biological chemistry. 2007; 282:37378-37388.

49. Chen X, Overcash R, Green T, Hoffman D, Asch AS, RuizEchevarria MJ. The tumor suppressor activity of the transmembrane protein with epidermal growth factor and two follistatin motifs 2 (TMEFF2) correlates with its ability to modulate sarcosine levels. The Journal of biological chemistry. 2011; 286:16091-16100.

50. Chen X Ruiz-Echevarria MJ. TMEFF2 modulates the AKT and ERK signaling pathways. International journal of biochemistry and molecular biology. 2013; 4:83-94.

51. Quayle SN, Sadar MD. A truncated isoform of TMEFF2 encodes a secreted protein in prostate cancer cells. Genomics. 2006; 87:633-637.

52. de Launoit Y, Baert JL, Chotteau-Lelievre A, Monte D, Coutte L, Mauen S, Firlej V, Degerny C, Verreman K. The Ets transcription factors of the PEA3 group: transcriptional regulators in metastasis. Biochimica et biophysica acta. 2006; 1766:79-87.

53. Shaikhibrahim Z, Lindstrot A, Ellinger J, Rogenhofer S, Buettner R, Perner S, Wernert N. The peripheral zone of the prostate is more prone to tumor development than the transitional zone: is the ETS family the key? Mol Med Rep. 2012; 5:313-316.

54. Barrett T, Wilhite SE, Ledoux P, Evangelista C, Kim IF, Tomashevsky M, Marshall KA, Phillippy KH, Sherman PM, Holko M, Yefanov A, Lee H, Zhang N, et al. NCBI, GEO. archive for functional genomics data sets-update. Nucleic acids research. 2013; 41:D991-995.

55. Tomlins SA, Mehra R, Rhodes DR, Cao X, Wang L, Dhanasekaran SM, Kalyana-Sundaram S, Wei JT, Rubin MA, Pienta KJ, Shah RB, Chinnaiyan AM. Integrative molecular concept modeling of prostate cancer progression. Nature genetics. 2007; 39:41-51.
56. True L, Coleman I, Hawley S, Huang CY, Gifford D, Coleman R, Beer TM, Gelmann E, Datta M, Mostaghel E, Knudsen B, Lange P, Vessella R, et al. A molecular correlate to the Gleason grading system for prostate adenocarcinoma. Proceedings of the National Academy of Sciences of the United States of America. 2006; 103:10991-10996.

57. Long Q, Xu J, Osunkoya AO, Sannigrahi S, Johnson BA, Zhou W, Gillespie T, Park JY, Nam RK, Sugar L, Stanimirovic A, Seth AK, Petros JA, et al. Global transcriptome analysis of formalin-fixed prostate cancer specimens identifies biomarkers of disease recurrence. Cancer research. 2014; 74:3228-3237.

58. Erho N, Crisan A, Vergara IA, Mitra AP, Ghadessi M, Buerki C, Bergstralh EJ, Kollmeyer T, Fink S, Haddad Z, Zimmermann B, Sierocinski T, Ballman KV, et al. Discovery and validation of a prostate cancer genomic classifier that predicts early metastasis following radical prostatectomy. PloS one. 2013; 8:e66855.

59. Schmittgen TD, Livak KJ. Analyzing real-time PCR data by the comparative C(T) method. Nat Protoc. 2008; 3:1101-1108.

60. Barros-Silva JD, Ribeiro FR, Rodrigues A, Cruz R, Martins AT, Jeronimo C, Henrique R, Teixeira MR. Relative $8 \mathrm{q}$ gain predicts disease-specific survival irrespective of the TMPRSS2-ERG fusion status in diagnostic biopsies of prostate cancer. Genes, chromosomes \& cancer. 2011; 50:662-671.

61. Kamburov A, Pentchev K, Galicka H, Wierling C, Lehrach H, Herwig R. ConsensusPathDB: toward a more complete picture of cell biology. Nucleic acids research. 2011; 39:D712-717.

62. Kamburov A, Stelzl U, Lehrach H, Herwig R. The ConsensusPathDB interaction database: update. Nucleic acids research. 2013; 41:D793-800.

63. Kamburov A, Wierling $\mathrm{C}$, Lehrach $\mathrm{H}$, Herwig $\mathrm{R}$. ConsensusPathDB--a database for integrating human functional interaction networks. Nucleic acids research. 2009; 37:D623-628. 